\title{
ADMINISTRACIÓN HOSPITALARIA EN LA "GESTION DEL RIESGO"
}

Trabajo de grado para optar por el título de

Especialistas en Auditoría en Salud

UNIVERSIDAD SANTO TOMÁS

DIVISION DE CIENCIAS ECONÓMICAS Y ADMINISTRATIVAS

FACULTAD DE ECONOMIA

AUDITORIA EN SALUD BOGOTA D.C. 


\title{
ADMINISTRACIÓN HOSPITALARIA EN LA "GESTION DEL RIESGO"
}

\section{Melissa Fernanda Lugo Jácome}

Doris Vergara Gómez

\author{
Director \\ Alejandra Valenzuela Cazés \\ Fisioterapeuta \\ Magíster en Salud Pública
}

\author{
UNIVERSIDAD SANTO TOMÁS \\ DIVISION DE CIENCIAS ECONÓMICAS Y ADMINISTRATIVAS \\ FACULTAD DE ECONOMIA \\ AUDITORIA EN SALUD \\ BOGOTA D.C. \\ 2018
}




\section{INDICE}

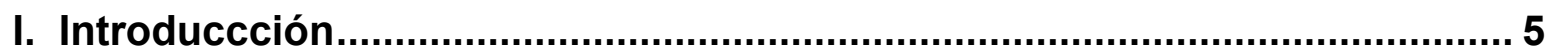

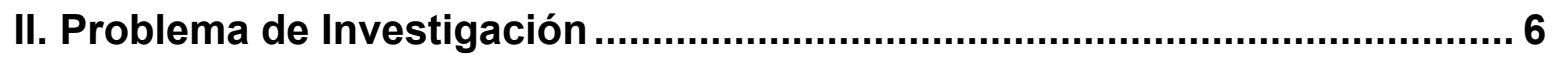

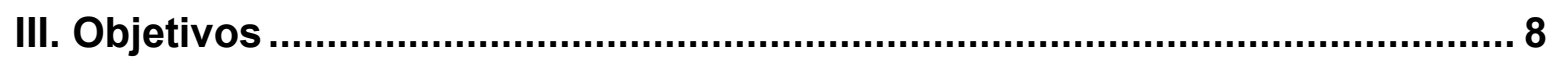

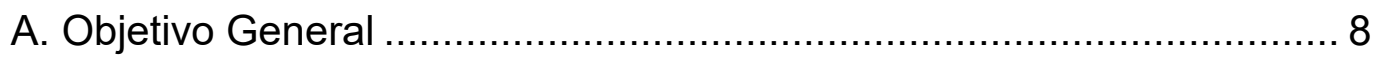

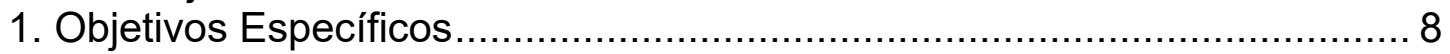

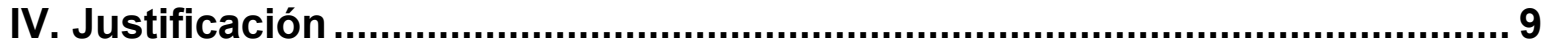

V. Elementos Conceptuales ....................................................................... 11

A. Administración Hospitalaria o Administración en Salud .............................. 11

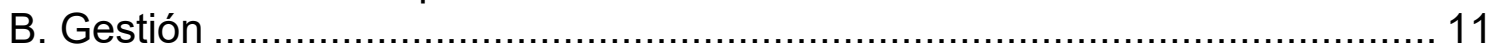

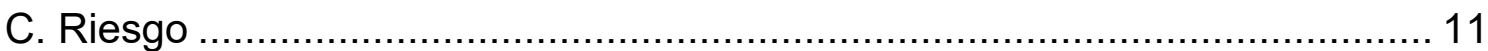

D. Gestión del riesgo.................................................................... 12

E. Administración hospitalaria en la gestión del riesgo en Instituciones de salud de algunos países de América Latina (Revisión de Artículos) ................. 12

1. Colombia: Implementación del Sistema de Administración de Riesgos SAR por Entidades Promotoras de Salud del Régimen Contributivo en Colombia 2008-2011 ..................................................................... 12

2. España - Alicante. Hospital Universitari Sant Joan d'Alacant: Gestión de riesgos desde la visión de la Gestión Hospitalaria ................................ 16

a. Papel de la Gestión Hospitalaria sobre el control de los efectos adversos................................................................................... 17

3. España - Ciudad Real. Complejo Hospitalario de Ciudad Real: Puesta en marcha de una Unidad de Gestión de Riesgos Clínicos Hospitalaria....... 18

a.Objetivos de la Unidad de gestión de riesgos ........................... 19

b. Fases de la puesta en marcha de la Unidad de Gestión............. 21

c. Aplicación práctica de la Unidad de riesgos en el Servicio de

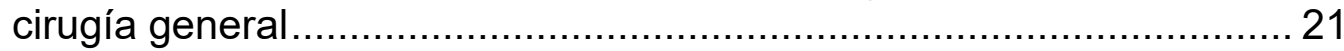

4. Argentina - Buenos Aires. Hospital Materno Infantil Ramón Sardá: Importancia de la Gestión de Seguridad en el Laboratorio Clínico de un Hospital Materno Infantil ................................................................. 22

a. Programa de Gestión de Riesgos ........................................ 22

5. CHILE - Concepción: Institución de Salud Previsional - ISAPRE Metodología de gestión de riesgo para procesos en una Institución Salud

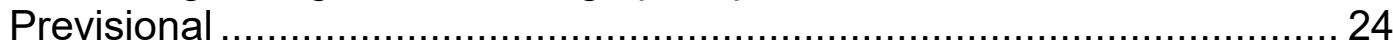

a. Metodología de Gestión de Riesgo para procesos críticos ........ 25

6. México: Evaluación del riesgo para la seguridad del paciente en



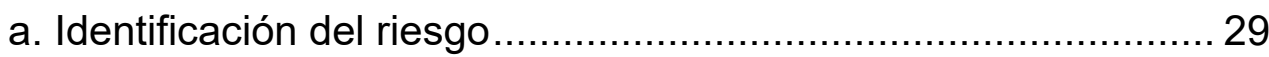

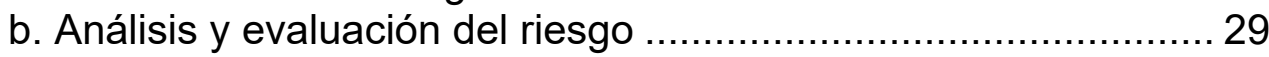

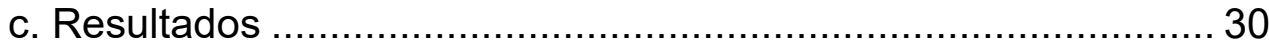




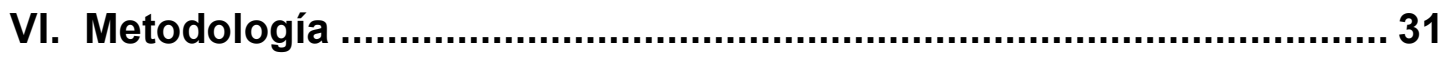

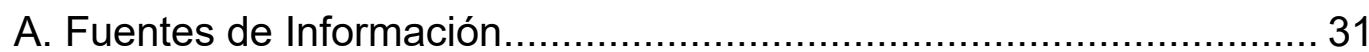

1. Clasificación según el grado de Información.................................. 31

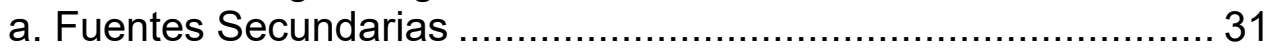

b. Fuentes Terciarias o Generales .......................................... 32



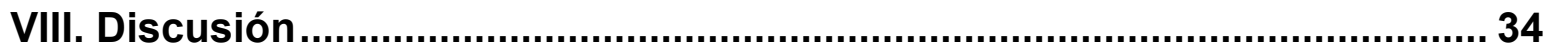

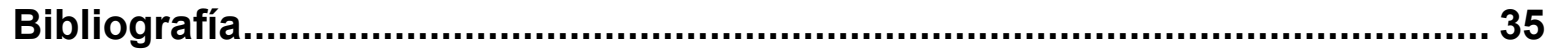

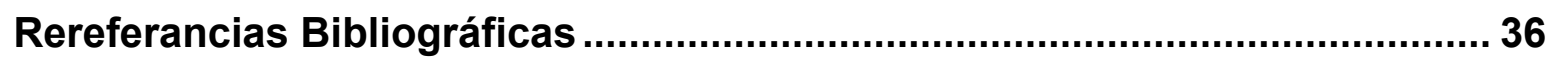




\section{INTRODUCCION}

La gestión del riesgo en salud constituye una herramienta útil para la gestión eficiente de los recursos, que permite contrarrestar el elevado riesgo y la incertidumbre de las actividades propias de este sector con el fin de generar valor, mejorar el desempeño y garantizar la supervivencia de la empresa en tiempos adversos, minimizando las pérdidas y maximizando las ganancias.

En Colombia, el concepto de gestión del riesgo en salud comienza a tenerse en cuenta mucho después de haberse desarrollado y aplicado en los sectores financiero, bancario y asegurador. El sector salud tiene similitudes con el sector financiero, lo cual hace posible aplicar los principios y las técnicas que se han desarrollado para este último.

Es por esto, que las empresas buscan conocer el nivel de riesgo en el que están incurriendo en las diferentes áreas, tanto operativas como estratégicas de la Organización. Es así como la normatividad nacional e internacional desde principios de los años noventa, ha definido la importancia de implementar este tipo de herramientas de control que permiten un mejor entendimiento de los procesos, anticiparse a los diferentes tipos de riesgo y facilitar la toma de decisiones gerenciales.

Dentro de la normatividad internacional se destacan el sistema Basilea I, II y III para riesgos financieros, el cual propone como beneficios globales: crear incentivos para mejorar los procedimientos de evaluación de riesgos, mejorar el sistema de gobierno corporativo, implementar nuevos modelos internos de evaluación de riesgo, lograr cambios culturales desde la alta dirección e innovar las herramientas de información con grandes inversiones en tecnología. Todo lo anterior, conlleva a un importante reordenamiento del sector, estos aspectos se encuentran reflejados en la normatividad adoptada por el Gobierno Colombiano y son aplicables tanto al sector financiero como al sector salud. 


\section{PROBLEMA DE INVESTIGACION}

A lo largo del tiempo, los países ubicados en Europa y de América Latina han atravesado por una serie de cambios demográficos, epidemiológicos, ambientales, económicos y sociales, entre otros, por ejemplo, hay un aumento considerable de las migraciones debidos a problemas políticos y sociales de algunos países, los cuales aumentan los índices de pobreza, de transmisión de enfermedades, entre otras situaciones y por ende el aumento en la demanda de servicios de salud, lo que ha llevado a que los diferentes gobiernos y sus instituciones creen y mejoren las políticas en materia de salud, incluyendo la administración del riesgo, sin embargo aún existen falencias en este tema.

Con respecto a Alburquerque, Artaza, Antunes, Arteaga, Et al (2001) mencionan que los riesgos en los hospitales aumentarán en el futuro, tanto para las personas que trabajan en ellos (riesgos tan diversos como contagios, aumento demandas legales), como para las que reciben atención (infecciones cruzadas, efectos indeseados o colaterales de los mismos procesos asistenciales). Y más aún corre riesgo cuando se refiere al manejo de los bienes intangibles, como la información, historias clínicas, el conocimiento científico, la creatividad de los profesionales sanitarios, la imagen de la institución o el prestigio ante la población. Además para profundizar en el concepto sobre la gestión de riesgos, por tanto, es un complemento de la gestión general y su objetivo consiste en disminuir el daño o la pérdida que se pueda causar a las personas, a la infraestructura, a la tecnología o a intangibles como consecuencia de comportamientos ineficientes, incorrectos, no éticos e incluso fraudulentos (1).

Es así como en las últimas décadas, diversos países de Europa y de América Latina, han trabajado en sus instituciones de salud en el manejo del riesgo, Pardo y Cols (2014) mencionan que las primeras actuaciones estuvieron dirigidas a proteger a los profesionales y a las organizaciones de las consecuencias económicas de las reclamaciones por la irresponsabilidad sanitaria.

Con referencia a lo anterior, por ejemplo, estos autores también refieren que Estados Unidos se constituyó unidades de gestión de riesgos en los hospitales, donde sus objetivos primordiales fueron la investigación de los incidentes, la tramitación de reclamaciones o la supervisión de las causas de litigio (2). Posteriormente, se comenzó a trabajar en la mejora de la seguridad del paciente, tema que en la actualidad tiene un fuerte impacto en las políticas de salud a nivel mundial.

De igual forma, existen diversos problemas al momento de realizar un análisis de los riesgos a nivel hospitalario; sin embargo, los investigadores Pardo, Navarro, Arguedas, Albeniz y Morón (2014) realizaron un estudio en España, 
específicamente en el Servicio Madrileño de Salud - SERMAS, tomando 31 de 33 unidades funcionales de gestión de riesgos sanitarios en el año 2011, las cuales pertenecían a hospitales de diversos niveles de complejidad encontrando que aún existen barreras en los sistemas de gestión de riesgos, tales como; falta de tiempo de los profesionales para mejorar la seguridad del paciente, el déficit de cultura, escasa difusión para el personal y falta de formación para los directivos, escasa motivación, resistencia al cambio, falta de recursos económicos, materiales y humanos, falta de herramientas o de incentivos, falta de liderazgo de directivos, falta de notificación de incidentes y falta de liderazgo en los servicios (3).

Por todo lo anterior, se evidencia que aún hay mucho camino por trabajar en la gestión del riesgo desde la parte administrativa, a pesar de que existan políticas, protocolos y demás sistemas en pro de reducir los riesgos sobre todo los centrados en el paciente, también se requiere que las instituciones de salud desde lo administrativo centren su atención en trabajar y crear sistemas de gestión de riesgo integrales, es decir con un enfoque sistemático, que trabaje todos los riesgos a los que puede estar expuesta e involucre de manera activa a todos sus miembros y no se continúe administrando de manera fragmentada.

Cada país y sus instituciones de salud, realizan un manejo del riesgo de manera diferente teniendo en cuenta sus propios modelos de salud y la organización de las instituciones de salud, no se puede decir que existe un sistema de gestión de riesgo de un país más importante que otro, por lo cual es fundamental conocer y profundizar en como la dirección o la gerencia administran el riesgo a nivel hospitalario, y tener referentes para el mejoramiento de los sistemas de gestión de riesgo de las instituciones colombianas. 


\section{OBJETIVOS}

\section{I.A. Objetivo General}

Describir la administración hospitalaria en algunos países de América Latina y España con respecto a la implementación de los sistemas de gestión del riesgo en sus instituciones de salud.

\section{I.A.1. Objetivos Específicos}

- Identificar los sistemas de riesgos concernientes en la administración hospitalaria.

- Establecer un cuadro comparativo de diferencias del sistema de riesgos relacionados con la administración de cada país.

- Realizar una propuesta de sistema de riesgos ideal básica para la administración hospitalaria. 


\section{JUSTIFICACIÓN}

Al tratar el tema de salud se puede estudiar desde muchas perspectivas, desde lo netamente biológico individual, social, epidemiológico, ambiental, etc, cualquiera que sea el enfoque es muy importante para determinar la calidad de vida de las personas y las sociedades, ya que tiene un impacto en la economía y es un indicador importante de progreso en un país, por ese motivo se observa la tendencia a un mayor interés por parte de los países de los países de Europa y América Latina en mejorar sus sistemas de salud, a través de la creación e implementación de políticas para que las instituciones de salud tengan una base para el manejo tanto administrativo como operacional.

En este sentido, es necesario definir el concepto de riesgo, la Organización Mundial de la Salud - OMS(4) en uno de sus informes sobre la salud mundial lo define como la probabilidad de un resultado sanitario adverso, o un factor que aumenta esa probabilidad, al orientar este concepto al entorno hospitalario, se encuentra que tanto pacientes, visitantes, y todo el personal que labora en una institución de salud están expuestos a un número ilimitado de riesgos y es en este punto donde la gestión del riesgo juega un papel muy importante.

En los inicios de los procesos investigativos de la gestión del riesgo o el risk management, en Estados Unidos inició su desarrollo en los años cincuenta, orientado a las pólizas y los seguros y a mediados de los años setenta en el tema sanitario, según el Australian/New Zealand Standard menciona que la gestión de riesgos es el término que se aplica a un sistema lógico y sistemático de identificar, analizar, valorar, tratar, hacer el seguimiento y comunicar los riesgos asociados a cualquier actividad, función o proceso, de manera que se posibilite a las organizaciones minimizar las pérdidas y maximizar las oportunidades (5).

Por lo tanto, autores como Martínez y Ruiz (2001), definen la gestión del riesgo en el ámbito de salud como un conjunto de actividades destinadas a minimizar el riesgo de que se produzca un efecto adverso durante la asistencia, o a disminuir sus consecuencias negativas; constituye un conjunto de medidas de autoprotección, que buscan prevenir o evitar las amenazas reales o potenciales de pérdidas económicas debidas a accidentes, lesiones o negligencia médica (6).

Partiendo de estos conceptos, se determina que un sistema de gestión de riesgos es una herramienta indispensable en el manejo administrativo de una institución de salud, ya que como lo menciona Martínez y Ruiz (2001), busca disminuir el coste de todos los riesgos asistenciales y no asistenciales, para ello se pretende obtener una praxis sanitaria y sistemas de trabajo más seguros para los pacientes y los profesionales; de igual forma se tiene como objetivo propiciar que el conocimiento trascienda a medida que surjan las circunstancias de acuerdo al contexto 
administrativo en las instituciones de salud que rodean una gama de actividades por parte de los trabajadores; esto con el fin de generar medidas de control de la calidad en busca de mejorar los estándares de atención, siendo la reducción de costos un subproducto de su actividad (7).

Se puede decir entonces, que un sistema de gestión de riesgos es una pieza fundamental en todos los ámbitos de una Institución de salud, ya que su implementación no solamente tiene impacto en los pacientes, sino también en el personal de salud, en el manejo adecuado de todos los equipos e infraestructura, en la parte económica, financiera, investigativa, en la aplicación de políticas, guías, protocolos, procesos, en el área legal, en el prestigio, el renombre, la adherencia y la fidelización de los usuarios, entre otros.

A su vez, también es importante conocer la experiencia de otros países en cuanto al desarrollo de los sistemas de gestión de riesgos en las Instituciones hospitalarias, para conocer los diversos enfoques que se pueden usar, ampliar el campo de conocimiento y tener en cuenta las mejores prácticas que puedan servir como guía para orientar ciertos procesos de los sistemas de gestión de riesgos en Colombia. 


\section{ELEMENTOS CONCEPTUALES}

A continuación, se describen algunos conceptos básicos para contextualizar el tema:

\section{V.A. Administración Hospitalaria o Administración en Salud}

La administración en salud o administración sanitaria es la ciencia social y técnica relacionada con la planificación, organización, dirección y control de las empresas públicas y privadas del sector salud, mediante la optimización de recursos financieros, tecnológicos y humanos. La administración de servicios de salud es una de las disciplinas de la salud pública. Su función es la integración eficiente y efectiva de los recursos humanos, físicos y económicos para lograr una atención óptima de servicios de salud al paciente (8).

\section{V.B. Gestión}

La palabra gestión proviene del latín gestio, y hace la referencia a la administración de recursos, sea dentro de una institución estatal o privada, para alcanzar los objetivos propuestos por la misma (9).

La gestión es una guía para orientar la acción, previsión, visualización y empleo de los recursos y esfuerzos a los fines que se desean alcanzar, la secuencia de actividades que habrán de realizarse para logar objetivos y el tiempo requerido para efectuar cada una de sus partes y todos aquellos eventos involucrados en su consecución (10).

Casassus (2000) define también la gestión como una capacidad de generar una relación adecuada entre la estructura, la estrategia, los sistemas, el estilo, las capacidades, la gente, y los objetivos superiores de la organización considerada. O dicho de otra manera, la gestión es la capacidad de articular los recursos de que se disponen de manera de lograr lo que se desea (11).

\section{V.C. Riesgo}

Riesgo es la posibilidad de que suceda algún evento que tendrá un impacto sobre los objetivos institucionales o del proceso. Se expresa en términos de probabilidad y consecuencias (12).

El riesgo también se puede definir como la combinación de la probabilidad de un suceso y sus consecuencias. En todos los tipos de empresa existe un potencial de sucesos y consecuencias que constituyen oportunidades para conseguir beneficios (lado positivo) o amenazas para el éxito (lado negativo) (13). 


\section{V.D. Gestión del riesgo}

La gestión de riesgos es el proceso de planificación, organización, dirección y control de los recursos humanos y materiales de una organización, con el fin de reducir al mínimo o aprovechar los riesgos e incertidumbres de la organización (14).

En el contexto hospitalario, la gestión del riesgo se orienta igualmente a reducir los riesgos de afectación en las personas, recursos y procesos, identificando y controlando principalmente las amenazas y disminuyendo la vulnerabilidad frente a éstas (15).

\section{V.E. Administración hospitalaria en la gestión del riesgo en Instituciones de salud de algunos países de América Latina (Revisión de Artículos)}

A continuación se realiza una revisión de diez artículos de países como Colombia, España, Argentina y Chile donde los autores dan a conocer las estrategias que utilizan algunas instituciones de salud para la administración del riesgo, donde se extrae de manera general la información más relevante para esta revisión documental.

\section{V.E.1. Colombia: Implementación del Sistema de Administración de Riesgos -SAR por Entidades Promotoras de Salud del Régimen Contributivo en Colombia 2008-2011}

El tema de la gestión del riesgo tanto a nivel nacional e internacional, en las últimas décadas ha cobrado importancia para todo tipo de organizaciones, teniendo en cuenta la normatividad internacional, el Gobierno Colombiano y sus instituciones han establecido una serie de normas sobre la administración del riesgo.

La gestión del riesgo tiene como finalidad buscar el equilibrio apropiado entre el reconocimiento de oportunidades para obtener ganancia y reducir las pérdidas. También es un factor de éxito, que reduce tanto la probabilidad de fallo, como la incertidumbre y acerca de la consecución de los objetivos generales de la empresa (16).

A partir de la década de los noventa en Colombia se origina un desarrollo normativo importante en cuanto al aseguramiento en salud, partiendo de la Ley 100/93, donde aparecen actores como las EPS, encargadas de la afiliación y responsables de establecer mecanismos para la gestión del riesgo, luego surgen otras normas donde se exige que se debe aparte del riesgo en salud, se debe manejar el riesgo financiero, como requisito para la habilitación de las instituciones. 
En cuanto a las EPS, teniendo en cuenta varios referentes normativos, se establece mediante la Resolución 1740 de 2008 el Sistema de Administración para el Sector Salud (SAR), la cual lo define como el conjunto de procesos, procedimientos y tareas sistemáticas para planear, hacer, verificar y actuar frente al riesgo (17).

El artículo, presenta el SAR, así como los niveles de implementación alcanzados por los actores del SGSSS durante los últimos tres años, menciona que las Entidades Administradoras de Planes de Beneficios (EAPB), autorizadas para administrar el Régimen Contributivo corresponden a 24 entidades, 20 se presentaron de manera voluntaria para implementar el SAR, de las cuales 15 entidades cumplieron con los requisitos para implementar las tres primeras fases del SAR. En la primera fase, se presentaron 17 entidades, de las cuales 15 cumplieron los requisitos, en la segunda se presentaron 20 entidades que cumplieron en su totalidad con los requisitos para esta fase, en la tercera se presentaron 20 EAPB de las cuales 15 cumplieron con los requisitos (18).

El proceso de implementación del SAR, inicialmente la normatividad le dio competencia a la Superintendencia Nacional de Salud para la selección y aprobación de las firmas consultoras habilitadas para que cada EPS libremente escogiera la firma que realizaría la evaluación de la metodología e implementación del SAR en cada una de sus fases. (Ver Figura 1,2 y 3 ).

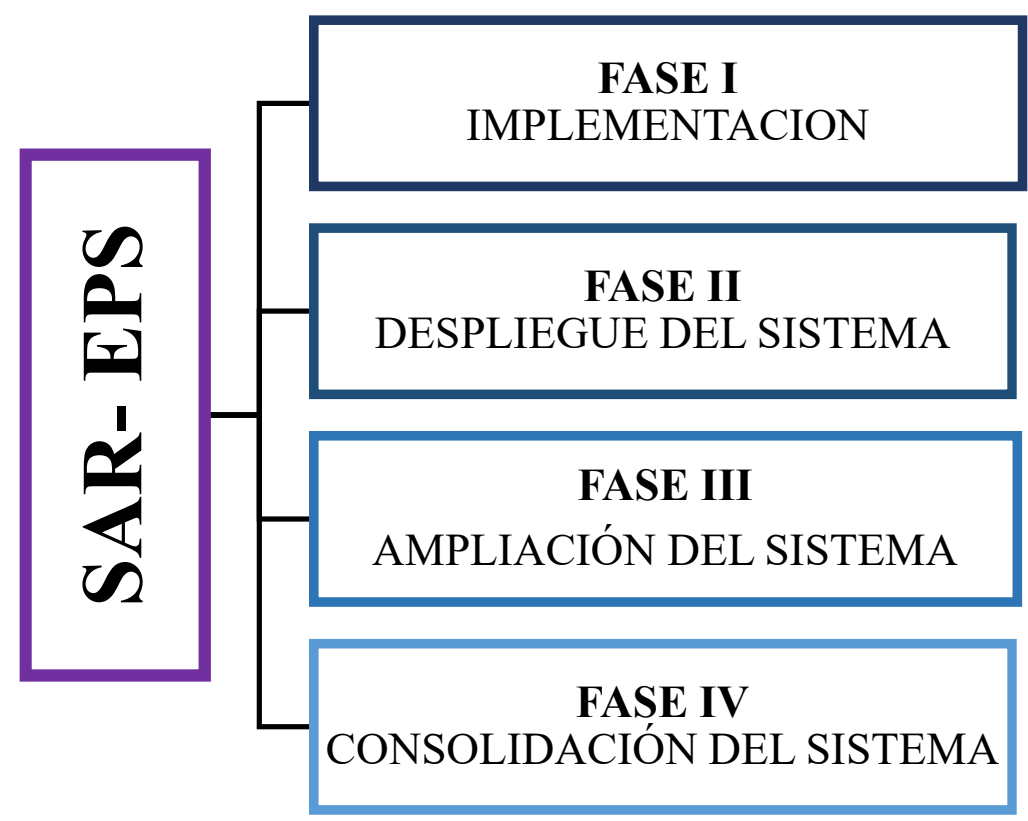

FIGURA 1.Fases de Implementación del Sistema de Administración de Riesgos- SAR Fuente: Elaboración propia 


\section{FASE I \\ $2008-2009$}

IMPLEMENTACION DEL SAR

En esta etapa significo cambios en la cultura organizacional, enfoque de los procesos y procedimientos hacia la identificación y control de los riesgos en salud.

- Formulación de políticas de implementación, determinar objetivos sobre el alcance de la gestión de riesgos, conformación de equipos, establecimiento de metodologias, adecuación del sistema de información y despliegue y socialización del sistema en los diferentes niveles de la organización.

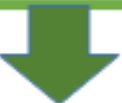

FASE II

$2009-2010$

\section{DESPLIEGUE DEL SISTEMA}

En esta etapa implico la identificación y priorización de los riesgos generales en salud, asi como el establecimiento de indicadores cualitativos frente al impacto de estos riesgos y de la gestión individualizada del riesgo.

- Lograr difusión interna y externa de los objetivos, medición de avances a partir de actividades ya existentes y ampliándolas a grupos priorizados de riesgos a los cuales se debe llegar a la gestión individualizada del riesgo, inicio de esquemas cualitativos de medición del impacto.

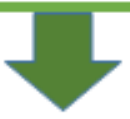

FIGURA 2. Descripción de las fases del Sistema de Administración de Riesgos Fuente: Elaboración propia 


\section{FASE III}

$2010-2011$

AMPLIACION DEL SISTEMA

Esta etapa permite el inicio de la consolidación del sistema dado que avanza a mediciones cuantitativas de los impactos de la gestión de riesgos en salud, además involucra las áreas operativa y financiera dentro del mapa de priorización para definir herramientas de control para estos.

- Evaluar el ambiente interno frente al reconocimiento del sistema y el avance del plan de implementación original,

establecer la gestión de riesgos compartidos, definir el Mapa de riesgos a partir del mapa de Procesos.

- Área de salud: Medición cuantitativa de riesgos priorizados, proporción de población afiliada por equipo de riesgo priorizado, seguimiento al impacto en la gestión de riesgos, medición de impacto de intervenciones.

Área Operativa: Identificar riesgos en los diferentes tipos de gestión; de planeación, comercial y operaciones, de información al usuario y tecnológica, riesgo legal y reputacional y de riesgos con la seguridad del paciente

Área Financiera: Identificar riesgo estratégico, de mercado y flujo de caja, de crédito de deudores, análisis del portafolio de inversiones, determinar requerimiento de liquidez de la Entidad,

En esta etapa se espera que la mayoría de las entidades establezcan la trazabilidad en indicadores cuantitativos de gestión de riesgos en salud e inicien la medición del impacto de la misma. Además se asegure el despliegue de la implementación de controles medibles en el mapa de riesgos operativos y financieros.

Área de salud: Verificar la manera sistemática de aplicación del ciclo de planear, hacer, verificar y actuar frente el riesgo.

Área operativa: Documentar la gestión del riesgo existente en la organización.

Área de riesgos generales del negocio: Documentar el control de riesgo del mercado y de crédito propio del sector salud.

FIGURA 3. Descripción de las fases del Sistema de Administración de Riesgos Fuente: Elaboración propia 
Los principales criterios evaluados por fase se sintetizan en cuatro dimensiones de riesgo a saber:

1. Riesgos Generales en Salud (RGS).

2. Riesgos Individuales en Salud (RIS).

3. Riesgos Operativos (RO)

4. Riesgos Financieros y de Mercado (RFM)

La Gestión del Riesgo como cultura organizacional constituye un eje transversal e integrador en los diferentes procesos, tiene por objetivo garantizar el desarrollo y las condiciones óptimas de seguridad posible, donde la atención y las acciones desplegadas promuevan el mismo desarrollo del sector salud, involucrando etapas como la identificación, la priorización, la mitigación de los riesgos y la mejora del estado de salud de la población como impacto social. (19)

\section{V.E.2. España - Alicante. Hospital Universitari Sant Joan d'Alacant: Gestión de riesgos desde la visión de la Gestión Hospitalaria}

El Hospital Universitari Sant Joan d'Alacant orienta la gestión del riesgo a reconocer las posibles causas que generan los eventos adversos y establecen estrategias eficaces para su disminución. Los autores mencionan que los efectos adversos (EA) tienen varias implicaciones tanto en los resultados asistenciales ya que pueden afectar al paciente y su entorno, a los profesionales, a las instituciones de salud y al sistema de salud en sí debido a:

1. Aparición de resultados peores que los esperados, los cuales pueden tener secuelas temporales, permanentes o fatales.

2. Retraso en la resolución de casos, lo que lleva al aumento de la estancia y los costos.

3. Mala percepción de la calidad de la asistencia de los centros sanitarios, lo que afecta su posición, prestigio y competitividad.

4. Influyen en la disminución de la motivación de los profesionales, aparecen prácticas defensivas, en muchos casos incompatibles con la adecuada praxis médica.

5. Favorecen la pérdida de confianza en el sistema sanitario entre los usuarios.

Sumado a esto existen varias causas que motivan la aparición de eventos adversos:

1. Complejidad de los procesos: Las instituciones de salud están conformados por una serie de procesos complejos, están divididos en compartimentos organizados por servicios centrales y asistenciales $y$, a su vez en sistemas y especialidades, la estructura del personal es fragmentada, con una distribución jerarquizada en divisiones (médica, de enfermería), además hay una organización del trabajo con distintos horarios y turnos, lo que favorece la aparición de efectos adversos, ya que no existe una asistencia estructurada e integral. 
2. Evolución tecnológica: Los avances científicos a la vez que son precisos, son complejos y requieren que los profesionales tengan una formación específica y continua, es así como algunos eventos adversos se pueden producir por falta de un adecuado aprendizaje.

3. Dificultades en la declaración de casos: Cualquier efecto adverso que se presenta conlleva a una responsabilidad por el daño ocasionado, ya sea individual o patrimonial por la que puede exigirse una compensación, por estas razones, existe temor para reportar los efectos adversos, dadas las implicaciones económicas, penales y de sanciones disciplinarias 0 administrativas por parte de las instituciones de salud.

4. Ineficacia o ausencia de políticas de gestión de riesgos: Dentro del ámbito hospitalario, la gestión sanitaria además de realizar actividades a un buen costo, también debe tener en cuenta los indicadores que controlen los posibles inconvenientes que puedan darse en la parte asistencial y así minimizar los eventos adversos. (20)

\section{V.E.2.a. Papel de la Gestión Hospitalaria sobre el control de los efectos adversos}

Teniendo en cuenta las causas enunciadas anteriormente, los autores exponen que existen unos elementos clave para minimizar la aparición de eventos adversos desde la gestión hospitalaria (Ver Figura 4 y 5).

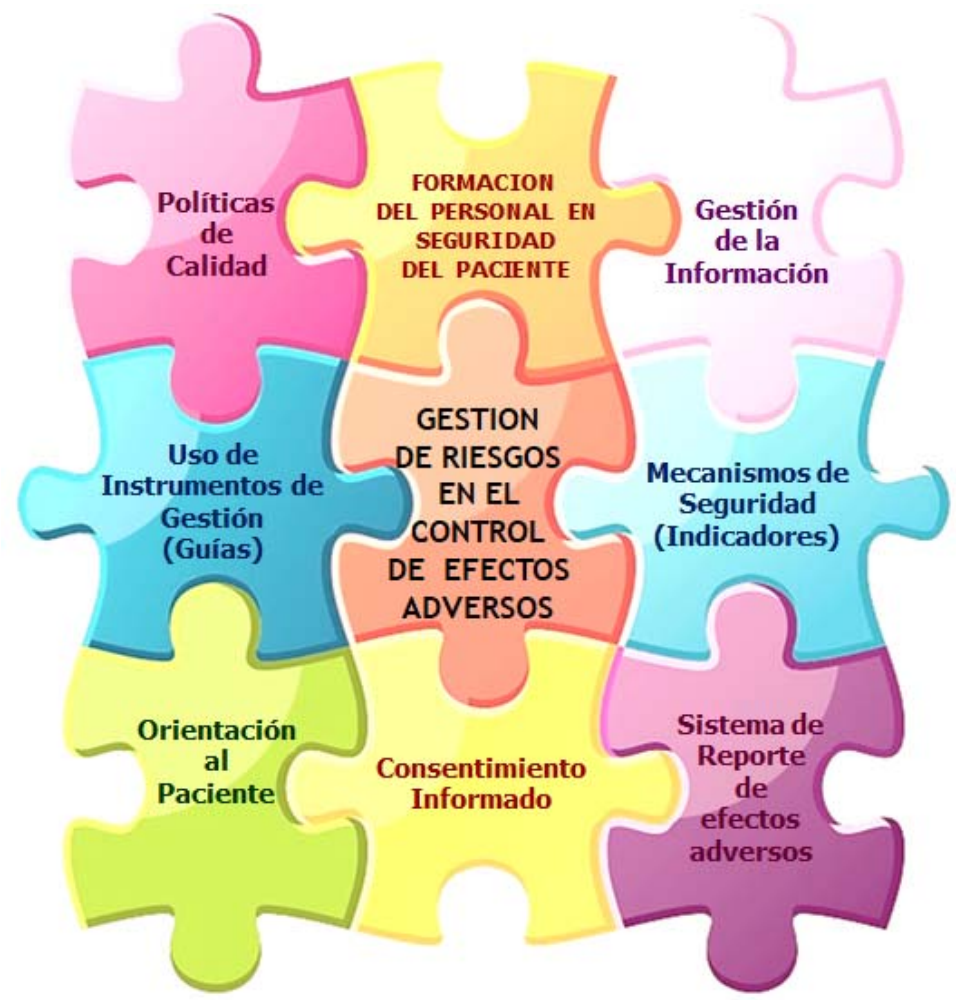

FIGURA 4. Elementos para la Gestión de Riesgos en el control de Efectos Adversos Fuente: Elaboración propia 
A continuación se describen brevemente cada uno de estos elementos:

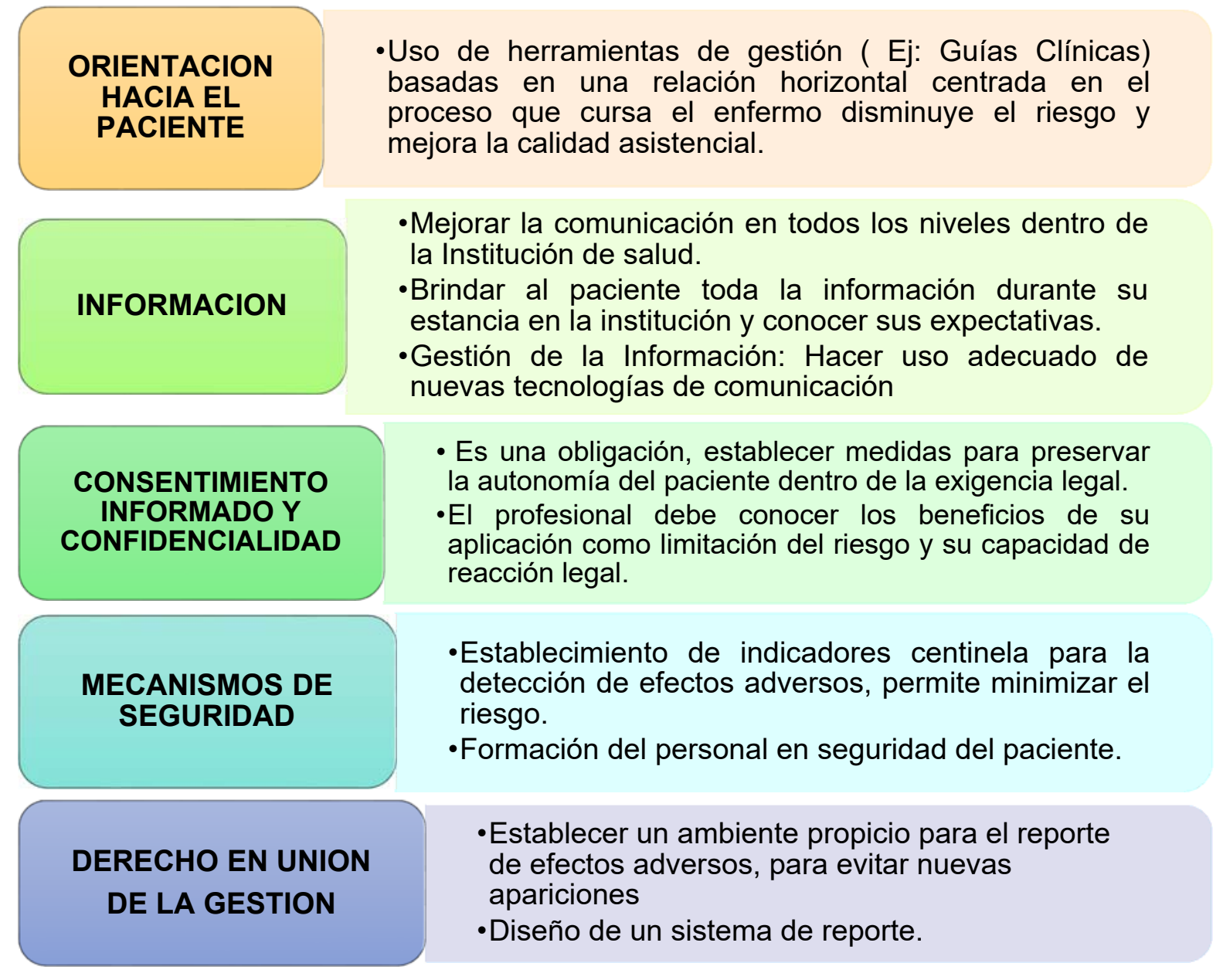

FIGURA 5. Descripción de Elementos para la Gestión del riesgo en el control de Efectos adversos. Fuente: Elaboración propia

\section{V.E.3. España - Ciudad Real. Complejo Hospitalario de Ciudad Real: Puesta en marcha de una Unidad de Gestión de Riesgos Clínicos Hospitalaria}

La gestión de riesgos clínicos mejora la calidad asistencial mediante el incremento de los niveles de seguridad en la atención a los pacientes intentando suprimir los errores evitables. La puesta en marcha de programas de gestión de riesgos clínicos requiere la creación de unidades dentro del organigrama hospitalario destinadas a canalizar las actividades relacionadas con la seguridad del paciente (21).

Los autores dan a conocer su experiencia en la creación y funcionamiento de una de las primeras Unidades de gestión de riesgos clínicos en Europa, ya que aún no existen políticas comunes sobre la seguridad del paciente, a diferencia de Estados 
Unidos y el Reino Unido quienes centran su política en salud en torno a la seguridad del paciente, específicamente en los errores humanos causados por el personal asistencial.

La gestión de riesgos clínicos se basa en la identificación, el análisis y la corrección de las causas que originan daño secundario al paciente tras el tratamiento médico. Es imposible evitar completamente el daño al paciente. Toda actividad humana conlleva un margen de error; sin embargo, sí es posible identificar, estudiar, controlar y minimizar ese daño; en definitiva, gestionar el riesgo que puede sufrir el paciente (22)

\section{V.E.3.a.Objetivos de la Unidad de gestión de riesgos}

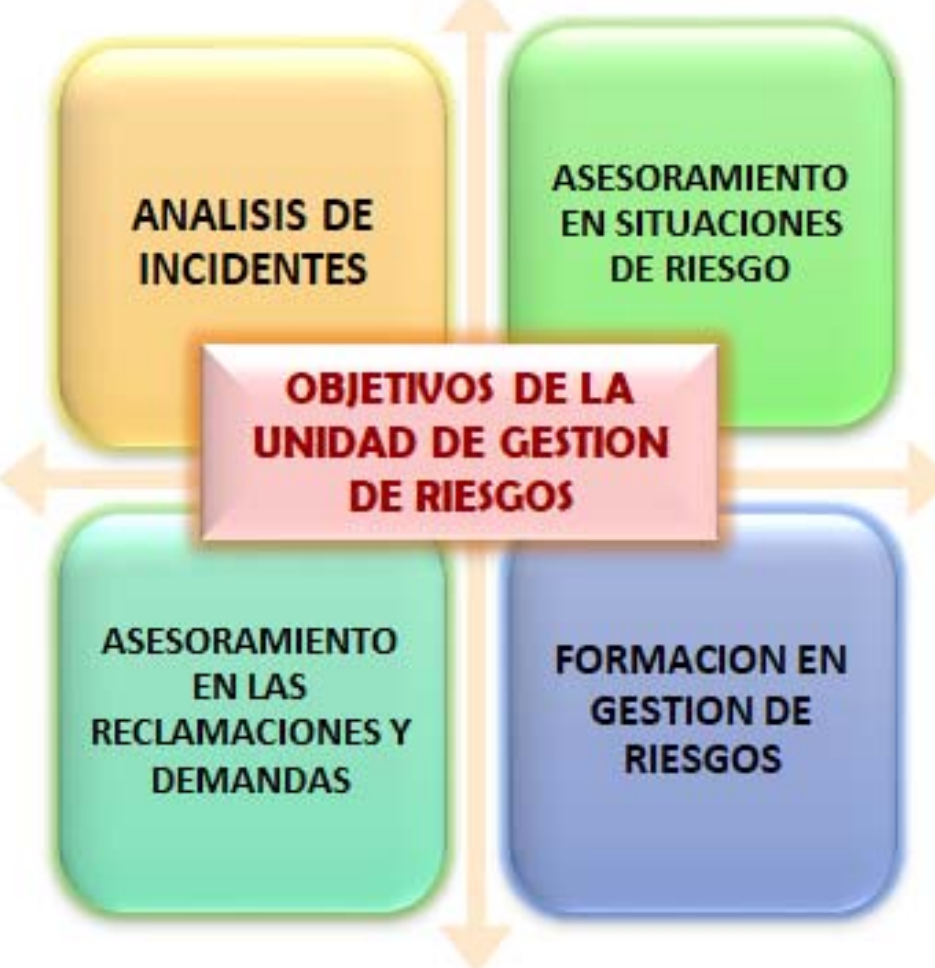

FIGURA 6. Objetivos de la Unidad de Gestión de Riesgos Fuente: Elaboración

Los autores Pardo, Et al (2005) describen cada uno de estos objetivos (23):

1. Análisis de Incidentes:

- Mejorar la calidad asistencial, mediante el perfeccionamiento de los niveles de seguridad de los pacientes, evitando en lo posible que se produzcan situaciones que originen un riesgo para su salud. 
- Desarrollo de un sistema de notificación de incidentes, donde luego de un análisis conjunto con el personal implicado, se emitirá un informe para la gerencia del centro en el que se propondrán las medidas correctoras que se consideren necesarias.

- Los servicios reciben una retroalimentación periódica de los incidentes de 2 formas: De forma inmediata a los jefes de servicio y, trimestralmente, se emitirá un informe con las medidas adoptadas. También de forma periódica, la unidad presentará al servicio en una sesión los incidentes, las medidas que se hayan puesto en marcha y su eficacia.

- Seguimiento de las medidas puestas en marcha y evaluación de su eficacia por parte de los responsables del servicio con la colaboración de la unidad.

\section{Asesoramiento en situaciones de riesgo:}

- Establecer el nivel de riesgo de cada servicio del hospital con la colaboración de sus miembros.

3. Asesoramiento en las reclamaciones y demandas:

- Análisis de las demandas y reclamaciones previas a cada servicio.

- Asesoramiento al personal sanitario ante la presentación de reclamaciones.

- Ofrecer asesoramiento a los servicios en los casos de demandas de responsabilidad patrimonial, para la elaboración de los informes y el análisis de los casos.

- Agilizar la tramitación de los casos de responsabilidad patrimonial para facilitar la puesta en contacto de los inspectores médicos con los servicios correspondientes.

- Mantener a los servicios al corriente del estado de las distintas demandas presentadas, tanto por vía penal como contencioso administrativo.

- Colaborar en la investigación de los incidentes por abuso o maltrato al personal sanitario, con el objetivo de evitar abusos verbales o físicos por parte de los familiares o pacientes. En caso de presentarse estas situaciones, la unidad de gestión de riesgos intentará llegar a un acuerdo de buena voluntad con los pacientes o familiares implicados y en caso de que esto no sea posible, o se repita el incidente, hará una propuesta de traslado del paciente a otro servicio o centro, para que siga recibiendo allí atención sanitaria

4. Formación en gestión de riesgos:

- Introducción a la gestión de riesgos para el personal sanitario y no sanitario del Complejo Hospitalario de Ciudad Real. Se ha diseñado un curso de $50 \mathrm{~h}$ que incluye 4 módulos que intentan instruir en las bases de la gestión de riesgos.

- Formación continuada en gestión de riesgos para el personal sanitario y no sanitario. 


\section{V.E.3.b. Fases de la puesta en marcha de la Unidad de Gestión}

Inicialmente para el funcionamiento de la Unidad de Gestión uno de los autores del artículo recibió formación en el tema y luego a partir de esto, se realizó una propuesta para la creación de un programa de gestión de riesgos clínicos en el Complejo Hospitalario de Ciudad Real y luego en los otros hospitales. Los autores Pardo, Et al (24) señalan las siguientes fases:

1. Acuerdo con la Gerencia:

- Para la implantación del Programa de Gestión de riesgos clínicos y la instauración de una Unidad de gestión de riesgos en el centro hospitalario.

- Aceptación de la Gerencia e inclusión de la Unidad en el contrato del año 2004.

2. Infraestructura

- Asignación de un espacio físico propio de la Unidad de gestión de riesgos.

3. Recurso Humano:

- Elección del personal permanente en la Unidad de gestión de riesgos: 3 cirujanos de Cirugía General y Aparato digestivo, supervisoras del área médica, quirúrgica, hospitalización y servicios centrales.

- Todos trabajan a tiempo parcial y con su labor asistencial, no se les compensa económicamente, pero se acordó con la gerencia conceder días libres por concepto de "dedicación especial"

4. Presentación y ejecución del Proyecto:

- Se presenta el proyecto a los jefes de servicio, supervisores de enfermería, jefes de servicio del centro hospitalario en sesión convocada por la gerencia y de forma voluntaria.

- Puesta en marcha del programa.

- Educación continuada al personal asignado a la Unidad de gestión de riesgos.

\section{V.E.3.c. Aplicación práctica de la Unidad de riesgos en el Servicio de cirugía general}

Los autores describen como ha sido la aplicación de la Unidad de riesgos en el servicio de Cirugía General, ya que este fue uno de los servicios pioneros en el desarrollo de la Unidad. (Ver Figura 7) 


\section{INTRODUCCION DE LA TERMINOLOGIA: \\ Uso de términos de gestión de riesgo en el servicio a través de sesiones y práctica}

REPORTE:

Puesta en marcha del reporte de incidentes.

DISCUSIÓN ABIERTA:

Análisis de las causas que originan reclamaciones o demanda y las medidas a adoptar para evitar su repetición.

\section{HISTORIAS CLINICAS:}

Historias clínicas con información más completa sobre el manejo

del paciente y la información transmitida al paciente y familiares

FIGURA 7. Aplicación de la Unidad de Gestión de Riesgos en el Servicio de Cirugía General del Complejo Hospitalario de Ciudad Real Fuente: Elaboración propia

\section{V.E.4. Argentina - Buenos Aires. Hospital Materno Infantil Ramón Sardá: Importancia de la Gestión de Seguridad en el Laboratorio Clínico de un Hospital Materno Infantil}

La gestión de seguridad en el Hospital Materno Infantil Ramón Sardá de Buenos Aires busca reducir al mínimo la probabilidad de que se produzcan incidentes no deseados, por lo cual tienen como objetivo demostrar la importancia de un Programa de Gestión de Prevención de Riesgos en el laboratorio clínico. Dentro de los laboratorios clínicos, existen diversos riesgos tales como la seguridad radioactiva, biológica, informática, de la calidad, etc., todos estos riesgos representan una amenaza en la interrupción de los procesos. Los autores definen el riesgo como una posibilidad o probabilidad de daños. (25)

Se puede decir que un laboratorio clínico está conformado tanto por el personal, los pacientes y los proveedores, donde se dan una serie de relaciones y procesos que requiere tener un control adecuado para minimizar los riesgos a los que puede estar expuesta esta población.

\section{V.E.4.a. Programa de Gestión de Riesgos}

Para planificar un Programa de Gestión de riesgos, los autores indican que se debe incluir:

1. Identificar, manejar y reducir los peligros/riesgos de todas las actividades desarrolladas en el Laboratorio Clínico. 
2. Adoptar un conjunto de acciones preventivas para eliminar y/o controlar los riesgos que se hayan detectado.

3. Capacitar asegurando la participación del personal para una mejora continua del desempeño ambiental, de seguridad y salud laboral.

4. Controlar la eficacia de las medidas preventivas adoptadas.

5. Integrar la acción preventiva en la gestión de la Organización.

6. Establecer una vigilancia adecuada de la salud de los trabajadores.

7. Desarrollar actuaciones ante situaciones de emergencia (26).

Para el desarrollo de este Programa de Gestión de riesgos, los autores resaltan la importancia que sea un sistema integrado, ya que cumple varias funciones tales como la duplicidad de procedimientos y normas, se dirigen las actividades dentro de la organización de forma integrada, se mejora la comunicación, por lo cual se hace necesario que la parte directiva de la organización participe y conozca el alcance y los beneficios de la implementación del Programa. Los autores proponen un plan de actividades preventivas (Ver Tabla 1).

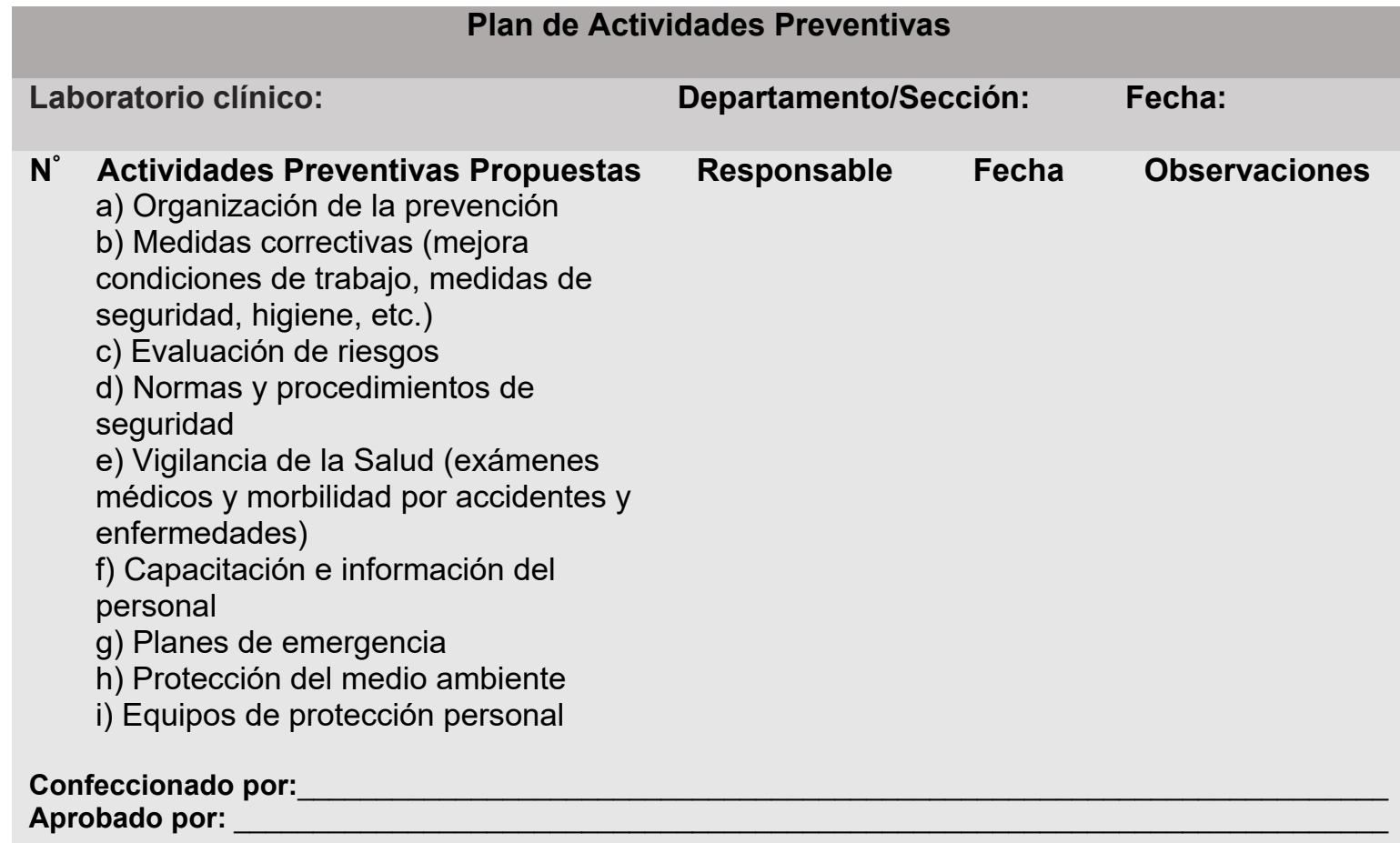

Tabla 1. Plan de Actividades Preventivas

FUENTE: Dear P, S. Importancia de la gestión de seguridad en el laboratorio clínico de un hospital materno infantil. Revista del Hospital Materno Infantil Ramón Sardá [Internet]. 2008. Disponible en: http://www.redalyc.org/articulo.oa?id=91227305. ISSN 1514-9838 


\section{V.E.5. CHILE - Concepción: Institución de Salud Previsional - ISAPRE Metodología de gestión de riesgo para procesos en una Institución Salud Previsional}

El artículo desarrolla una investigación en una Institución de Salud Previsional (ISAPRE) de Concepción, Chile, con el fin de implementar un sistema de gestión de riesgo para un conjunto procesos de interés de la Superintendencia de Salud. El objetivo del estudio es diseñar una metodología para introducir prácticas de gestión de riesgo en dicha institución (27).

La gestión de riesgo es una práctica de gestión que ayuda a predecir y manejar eventos que eviten la entrega de un servicio fuera de lo planificado, es "una forma eficiente de identificar oportunidades y evitar pérdidas y ayuda a mejorar el funcionamiento de las unidades de negocios de cualquier tipo de empresa, ya sea pública o privada, "todas las organizaciones, independientemente de su naturaleza, tamaño y razón de ser, están expuestas a diferentes riesgos que pueden poner en problemas su existencia; por lo tanto el objetivo de la gestión del riesgo es lograr que el proceso y sus controles garanticen que los riesgos están minimizados y que los objetivos de la organización van a ser alcanzados"(28).

Los autores realizaron una revisión de la literatura y describen diversas metodologías de gestión de riesgo a nivel gubernamental, financiero, municipal, sin embargo no encontraron estudios ni aplicaciones en la Industria de la Salud Previsional, por lo que diseñaron una Metodología de Gestión de Riesgo para procesos críticos, conformada por siete procesos teniendo en cuenta las diversas metodologías consultadas (Ver Figura 8).

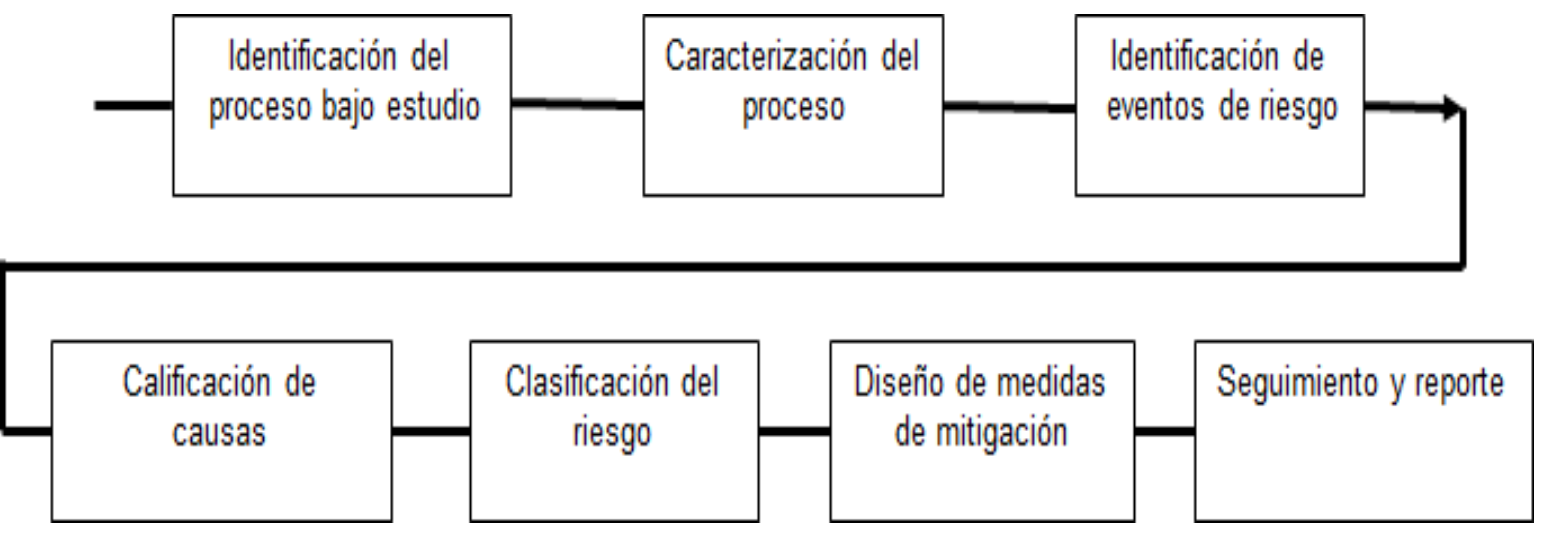

FIGURA 8. Propuesta de secuencia Metodológica para la Gestión del Riesgo

FUENTE: Torres N, C.Malta C,N.Zapata C, C. Aburto V, Víctor. Metodología de Gestión de Riesgo para procesos en una Institución de Salud Previsional. Revista Universidad, Ciencia y Tecnología [Internet]. 2015. Disponible en: $\underline{h t t p: / / w w w . s c i e l o . o r g . v e / s c i e l o . p h p ? s c r i p t=s c i ~ i s s u e t o c \& p i d=1316-~}$

$\underline{482120150002 \& \operatorname{lng}=e s \& n r m=i s O}$ 


\section{V.5.E.a. Metodología de Gestión de Riesgo para procesos críticos}

A continuación, los autores Torres, Malta, Zapata y Aburto (2015), realizan una descripción de cada una de las etapas y los instrumentos utilizados (29):

1. Identificación del Proceso bajo estudio: Esta etapa tiene la finalidad de definir el proceso que será objeto de la gestión de riesgo. Los procesos son los siguientes: Tramitación y pago de solicitudes de reembolso, mantención de contratos y cobranza de cotizaciones.

2. Caracterización del Proceso: Elaboración de una ficha (Ver Figura 9).

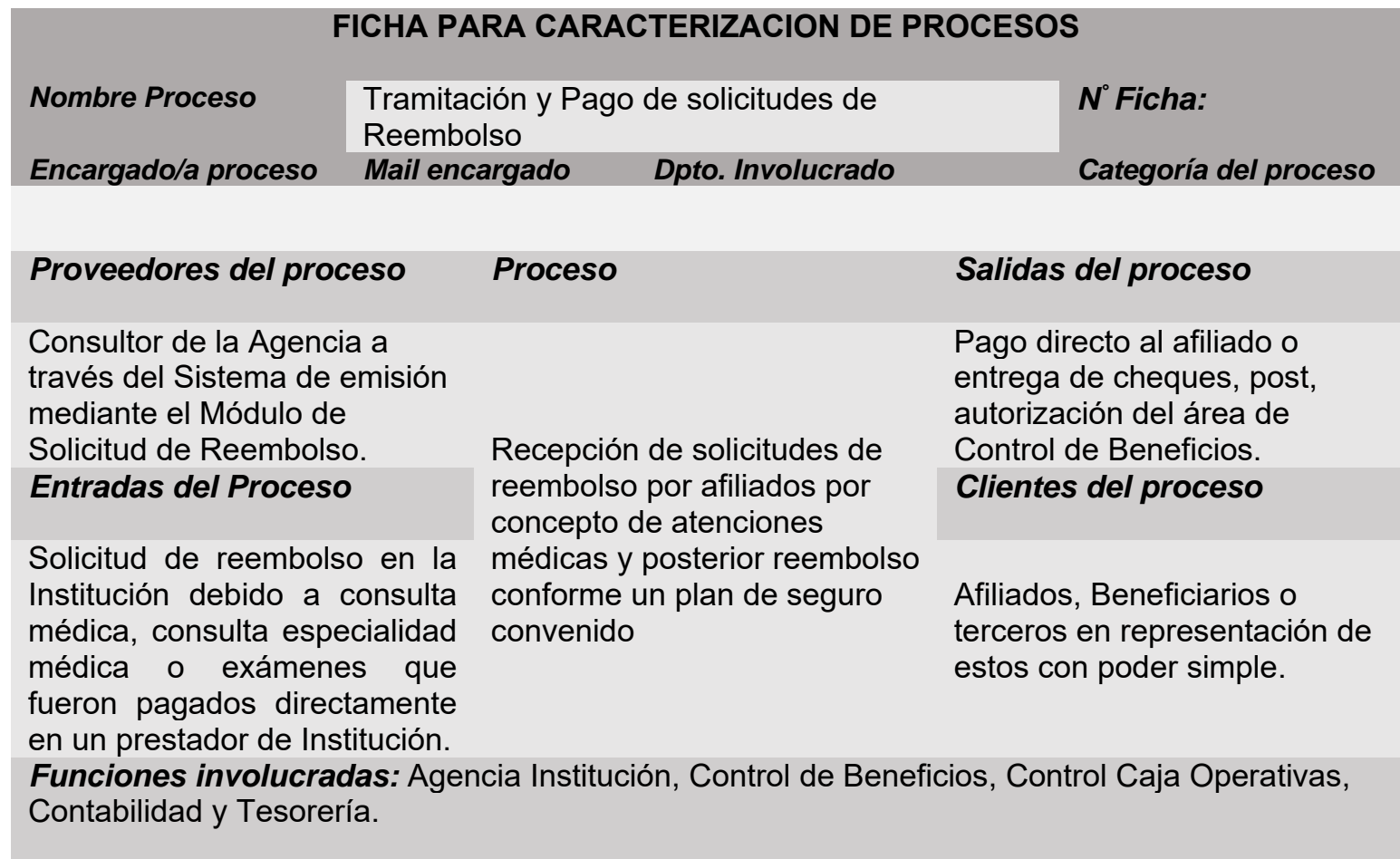

Tabla 2. Ficha para Caracterización de Procesos

FUENTE: Torres N, C.Malta C,N.Zapata C, C. Aburto V, Víctor. Metodología de Gestión de Riesgo para procesos en una Institución de Salud Previsional. Revista Universidad, Ciencia y Tecnología [Internet]. 2015. Disponible en: $\underline{h t t p: / / w w w . s c i e l o . o r g . v e / s c i e l o . p h p ? s c r i p t=s c i ~ i s s u e t o c \& p i d=1316-}$ $\underline{482120150002 \& \operatorname{lng}=e s \& n r m=i s O}$

3. Identificación de eventos de Riesgo: En esta etapa se reconocen los eventos riesgosos y las fallas que afectan el proceso de estudio, pueden afectar tanto al afiliado como a la Institución de Salud Previsional (Ver Tabla 2). 


\begin{tabular}{|c|c|c|}
\hline PROCESO & EVENTO DE RIESGO & AFECTADO \\
\hline Tramitación y & Posibilidad de reembolsar boletas falsas. & ISAPRE \\
\hline & Posibilidad de incumplir los plazos en el pago de & AFILIADO \\
\hline $\begin{array}{l}\text { Reembolso } \\
\text { Reembolitudes ae }\end{array}$ & reemidoisos ameriás & \\
\hline $\begin{array}{l}\text { Mantención de } \\
\text { Contratos }\end{array}$ & $\begin{array}{l}\text { Posibilidad de no otorgar la cobertura que corresponda } \\
\text { a un beneficiario y/o afectar la cotización pactada por } \\
\text { una modificación de contrato }\end{array}$ & AFILIADO \\
\hline \multirow{3}{*}{$\begin{array}{l}\text { Cobranza de } \\
\text { Cotizaciones }\end{array}$} & $\begin{array}{l}\text { Posibilidad de no poder reajustar el precio base de los } \\
\text { planes contratados por los afiliados. }\end{array}$ & AFILIADO \\
\hline & $\begin{array}{l}\text { Posibilidad de efectuar la cobranza de cotización a un } \\
\text { afiliado que no posee deuda }\end{array}$ & AFILIADO \\
\hline & $\begin{array}{l}\text { Posibilidad de no efectuar la cobranza de cotización a } \\
\text { un afiliado que si posee deuda }\end{array}$ & ISAPRE \\
\hline
\end{tabular}

Tabla 3. Identificación de Eventos de Riesgo

FUENTE: Adaptado de Torres N, C.Malta C,N.Zapata C, C. Aburto V, Víctor. Metodología de Gestión de Riesgo para procesos en una Institución de Salud Previsional. Revista Universidad, Ciencia y Tecnología [Internet]. 2015. Disponible en:

http://www.scielo.org.ve/scielo.php?script=sci issuetoc\&pid=1316-482120150002\&lng=es\&nrm=iso

4. Calificación de Causas: En esta etapa se califica la frecuencia e impacto de cada una de las causas de cada evento de riesgo recurriendo a la experiencia de las personas involucradas en cada caso. Se establecen las consecuencias, causas, frecuencia de falla, el impacto y el nivel de Riesgo.

\begin{tabular}{|c|c|c|c|c|c|c|}
\hline $\mathbf{N}$ & $\begin{array}{l}\text { Evento de } \\
\text { riesgo }\end{array}$ & Consecuencias & Causas & $\begin{array}{l}\text { Frecuencia } \\
\text { de falla }\end{array}$ & Impacto & $\begin{array}{c}\text { Nivel de } \\
\text { riesgo }\end{array}$ \\
\hline 1 & $\begin{array}{l}\text { Posibilidad de } \\
\text { reembolsar } \\
\text { boletas falsas } \\
\text { o robadas }\end{array}$ & $\begin{array}{l}\text { 1.Pérdida } \\
\text { económica } \\
\text { asociada al } \\
\text { pago del } \\
\text { reembolso }\end{array}$ & $\begin{array}{l}\text { Malas } \\
\text { prácticas de } \\
\text { los afiliados } \\
\text { y/o terceros } \\
\text { (A). }\end{array}$ & Posible & Moderado & Medio \\
\hline 2. & $\begin{array}{l}\text { Posibilidad de } \\
\text { reembolsar } \\
\text { boletas a } \\
\text { personas que } \\
\text { no }\end{array}$ & $\begin{array}{l}\text { 1. Reclamos por } \\
\text { parte del afiliado } \\
\text { por reembolsos } \\
\text { a personas que } \\
\text { no corresponde }\end{array}$ & $\begin{array}{l}\text { Suplantación } \\
\text { de la } \\
\text { identidad del } \\
\text { afiliado (B). }\end{array}$ & Posible & Moderado & Medio \\
\hline & corresponde & $\begin{array}{l}2 . \quad \text { Pérdida } \\
\text { económica } \\
\text { asociada al pago } \\
\text { del reembolso. }\end{array}$ & & $\begin{array}{l}\text { Poco } \\
\text { Posible }\end{array}$ & Menor & Bajo \\
\hline
\end{tabular}

Tabla 4. Calificación de las Causas en Cada Evento de Riesgo

FUENTE: Torres N, C.Malta C,N.Zapata C, C. Aburto V, Víctor. Metodología de Gestión de Riesgo para procesos en una Institución de Salud Previsional. Revista Universidad, Ciencia y Tecnología

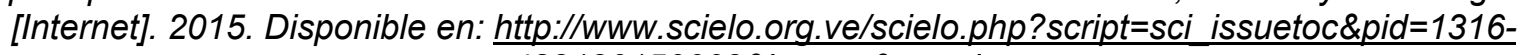
482120150002\&lng=es\&nrm=iso 
5. Clasificación de Riesgo: En esta etapa se reconoce el tipo de riesgo que se puede asociar con cada una de las causas que generan un evento de riesgo. Se aplica una matriz de riesgo.

6. Diseño de Medidas de Mitigación: Se establecen medidas para moderar, aplacar o suavizar los impactos de una causa de riesgo en función del nivel de riesgo asignado.

\begin{tabular}{|ll}
\multicolumn{1}{c}{ NIVEL DE RIESGO } & \multicolumn{1}{c}{ MEDIDAS A ADOPTAR } \\
Bajo & El riesgo se acepta y se debe controlar \\
Medio & $\begin{array}{l}\text { El riesgo se acepta, se debe investigar y } \\
\text { controlar }\end{array}$ \\
Alto y Muy Alto & $\begin{array}{l}\text { El riesgo se debe mitigar, a partir de } \\
\text { medidas correctivas o de mitigación, } \\
\text { además se debe controlar }\end{array}$ \\
\hline
\end{tabular}

Tabla 5. Medidas de Mitigación

FUENTE: Torres N, C.Malta C,N.Zapata C, C. Aburto V, Víctor. Metodología de Gestión de Riesgo para procesos en una Institución de Salud Previsional. Revista Universidad, Ciencia y Tecnología [Internet]. 2015. Disponible en: http://www.scielo.org.ve/scielo.php?script=sci issuetoc\&pid=1316$\underline{482120150002 \& \operatorname{lng}=e s \& n r m=i s o}$

7. Seguimiento y Reporte: En esta etapa se realiza el diseño de indicadores que afectan al usuario y a la institución.

Dentro de los resultados que los autores relatan se encuentra que el desarrollo de la Metodología involucro al personal directivo y técnico de la Institución, los resultados alcanzados aportan a la literatura de gestión del riesgo en este tipo de Instituciones, ya que hasta ahora es un concepto en desarrollo en este tipo de industria. A su vez, la metodología planteada rescata los aportes de varios instrumentos de riesgo, por lo cual es importante la revisión de la literatura y las experiencias de otro tipo de empresas (30).

\section{V.E.6. México: Evaluación del riesgo para la seguridad del paciente en establecimientos de salud}

La atención en instituciones de salud presenta riesgos implícitos para las personas que reciben la atención, la evaluación y gestión integral de riesgos aumenta la posibilidad de reducir los incidentes y eventos adversos y mejorar la seguridad de los pacientes. Existe diversidad de métodos para evaluar el riesgo en salud, lo que indica que no hay métodos únicos, ya que cada uno tiene fortalezas y debilidades, por lo que se hace necesario realizar una revisión documental, el reporte voluntario de eventos adversos y la evaluación de factores críticos del paciente (31). 


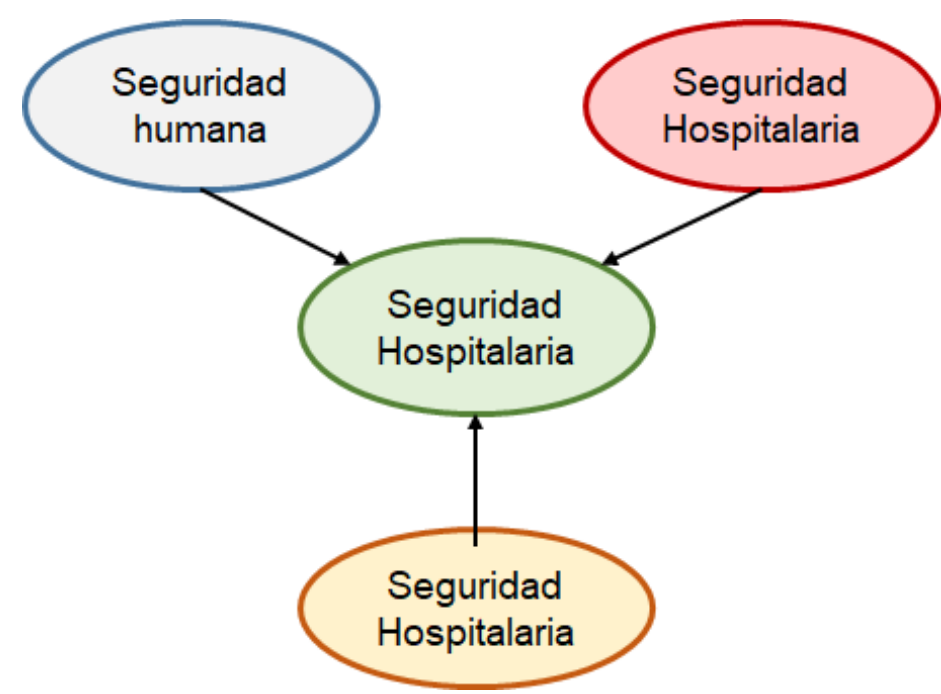

FIGURA 9. Categorías y niveles de análisis de la seguridad y su interrelación

FUENTE: Santacruz V, J. Hernández T, F. Fajardo D, G. Evaluación del riesgo para la seguridad del paciente en Establecimientos de Salud. Cirugía y Cirujanos [Internet]. 2010.

Disponible en: http://www.redalyc.org/articulo.oa?id=66220323009. ISSN 0009-7411

Los autores Santacruz, Hernández y Fajardo (2010) describen 4 categorías de seguridad (32) las cuales están relacionadas entre sí, y se detallan a continuación: (Ver figura 9)

1. Seguridad Humana: Tiene un objetivo cuantitativo, que es proporcionar seguridad en el acceso a los servicios de salud y el cualitativo darle seguridad de una atención con calidad.

2. Seguridad Hospitalaria: Debe brindar un nivel de protección a nivel de la infraestructura que le permita brindar servicios incluso en situaciones de desastre. También consiste en crear condiciones de seguridad para la vida de los pacientes, el personal y los visitantes, se usa el Índice de seguridad para medir riesgos estructurales y no estructurales.

3. Seguridad Clínica: Aquí se incluyen acciones sobre el diagnóstico, tratamiento, proceso de atención, ambiente, insumos, equipos y recurso humano.

4. Seguridad del Paciente: Es el resultado de la seguridad del sistema sanitario, donde se incluye todo lo relacionado con el uso y vigilancia de medicamentos, relación médico-paciente, aplicación de principios ética médica, buena praxis, uso adecuado de equipos y tecnologías, uso de listas de chequeo, entre otras.

En el desarrollo del estudio se diseñó un método para evaluar el riesgo hospitalario para la seguridad del paciente basado en modelos teóricos de la administración pública y sistemas de salud de países desarrollados. A continuación, los autores realizaron la descripción de cada una de las etapas, que para efectos del presente trabajo se presenta de manera resumida (33). 


\section{V.E.6.a. Identificación del riesgo}

Un grupo de la institución conformado por personal administrativo y asistencial identifico los factores de riesgo potenciales para la seguridad del paciente utilizando en enfoque sistémico de Donabedian, encontrando 18 factores de riesgo, distribuidos en los tres componentes del enfoque de sistemas (Ver Figura 10)

\begin{tabular}{|c|c|c|}
\hline $\begin{array}{l}\text { DE ESTRUCTURA } \\
\text { 1. Capacidad de Planeación- } \\
\text { Gestión } \\
\text { 2. Acceso al sistema de } \\
\text { atención } \\
\text { 3. Vulnerabilidad no } \\
\text { estructural y funcional } \\
\text { 4. Desarrollo del personal } \\
\text { 5. Calidad de los insumos } \\
\text { 6. Funcionalidad del } \\
\text { equipamiento }\end{array}$ & $\begin{array}{l}\text { DE PROCESO } \\
\text { 1. Documentación médica } \\
\text { 2. Prescripción de } \\
\text { medicamentos } \\
\text { 3. Comités hospitalarios } \\
\text { 4. Proyectos de } \\
\text { seguridad del paciente } \\
\text { 5. Registro de eventos } \\
\text { adversos } \\
\text { 6. Tiempo de espera y } \\
\text { atención diferida }\end{array}$ & $\begin{array}{l}\text { DE RESULTADOS } \\
\text { 1. Satisfacción de los usuarios } \\
\text { con la atención recibida } \\
\text { 2. Infecciones hospitalarias } \\
\text { 3. Reingreso al hospital } \\
\text { 4. Reintervenciones a } \\
\text { pacientes } \\
\text { 5. Mortalidad hospitalaria } \\
\text { 6. Capacidad resolutiva }\end{array}$ \\
\hline
\end{tabular}

FIGURA 10. Definición con enfoque sistémico de las variables de Evaluación del riesgo para la seguridad del paciente

FUENTE: Santacruz V, J. Hernández T, F. Fajardo D, G. Evaluación del riesgo para la seguridad del paciente en Establecimientos de Salud. Cirugía y Cirujanos [Internet]. 2010.

Disponible en: http://www.redalyc.org/articulo.oa?id=66220323009. ISSN 0009-7411

\section{V.E.6.b. Análisis y evaluación del riesgo}

En esta etapa se conformó un grupo de evaluadores y se utilizó un sistema de puntuación para calificar el riesgo de cada componente (Estructura, proceso, resultado).

- Puntuación $\leq 25 \%$-Riesgo crítico

- Puntuación de 26 a 50\%-Riesgo alto

- Puntuación de 51 a 75\%-Riesgo moderado

- Puntuación de 76 a 100\%-Riesgo bajo

El grupo decidió usar y adaptar el método denominado "siete pasos para la seguridad del paciente" utilizado en el Sistema Nacional de Salud Inglés, lo dejaron solo en 6 pasos que lo llamaron "Ciclo para la seguridad del paciente:

1. Evaluación del riesgo: Teniendo en cuenta las variables identificadas mediante el enfoque sistémico.

2. Priorización de Variables: Se ordenan por grado de riesgo.

3. Propuestas de Solución: Con el personal de salud se define un plan de acción.

4. Implementación de acciones: Llevar a cabo las acciones definidas en el plan. 
5. Monitoreo y Ajuste: Seguimiento permanente y evaluación para realizar ajustes.

6. Retroalimentación: Se realiza nuevamente una evaluación de las variables y si se logró su modificación.

\section{V.E.6.C. Resultados}

A través de la evaluación y el estudio de variables, se presenta en forma resumida los resultados obtenidos en cada uno de los componentes:

1. De Estructura: Obtuvo un $73,3 \%$ el cual representa un riesgo moderado, sugiriendo la necesidad de efectuar en el corto plazo acciones para mejorar la calidad de la infraestructura y funcionalidad de los equipos.

2. De Proceso: La calificación en conjunto fue de un $41.6 \%$ lo que indica un riesgo alto, ya que en el hospital no existe el informe sistematizado de eventos adversos, bajo desempeño en la calidad de los registros y prescripción de medicamentos.

3. De Resultado: El promedio general fue del $70 \%$, que lo ubica en un riesgo moderado, lo que sugiere que se deben crear oportunidades de mejora en algunas áreas del hospital.

La metodología desarrollada indica que es útil para evaluar y gestionar los riesgos relacionados con la atención médica hospitalaria, para estimular tanto a la dirección como al personal de salud en la necesidad de medir los riesgos y establecer un plan de acción para minimizarlos. 


\section{METODOLOGÍA}

Para la elaboración de este trabajo se realizó una revisión de la literatura, Hernández Sampieri y Cols (2006), menciona que la revisión de la literatura consiste en detectar, obtener y consultar la bibliografía y otros materiales que sean útiles para los propósitos del estudio, de donde se debe extraer y recopilar la información relevante y necesaria que atañe a nuestro problema de investigación (34).

El artículo de revisión es considerado como un estudio detallado, selectivo y crítico que examina la bibliografía publicada y la sitúa en cierta perspectiva Day RA (1994). Un artículo de revisión no es una publicación original y su finalidad es realizar una investigación sobre un tema determinado, en la que se reúnen, analizan y discuta la información relevante y necesaria qué atañe al problema de investigación que se desea abordar Sackett DL y Cols (1998). El objetivo fundamental del artículo de revisión es el de intentar identificar qué se conoce del tema, qué se ha investigado, así como conocer los avances más destacados que dicho tema ha tenido en un período de tiempo determinado y qué aspectos permanecen desconocidos (35).

\section{VI.A. Fuentes de Información}

Gallego y Junca (2009) mencionan que se puede entender fuente de información como toda huella o vestigio, testimonio y conocimiento legado por el discurrir de los hombres y mujeres a lo largo de la Historia. De ello se desprende que la fuente de información es todo lo que contiene información para ser transmitida o comunicada y que permite identificarse con el origen de la información. Merlo Vega (2002) propone entender las fuentes de información, en un sentido amplio, como el recurso empleado para satisfacer cualquier demanda de información, matizando que las fuentes pueden ser tanto documentos, como personas o instituciones (36).

\section{VI.A.1. Clasificación según el grado de Información}

Danhke (1989) distingue tres tipos básicos de fuentes de información para llevar a cabo la revisión de la literatura: las fuentes primarias, secundarias y terciarias, para el desarrollo de este trabajo se trabajaron las fuentes secundarias y terciarias.

\section{VI.A.1.a. Fuentes Secundarias}

Son compilaciones, resúmenes y listados de referencias publicadas en un área de conocimiento en particular (son listados de fuentes primarias). Es decir, reprocesan información de primera mano. Como ejemplos de fuentes secundarias tenemos: Las bibliografías, catálogos, repertorios, boletines de índices, sumarios y resúmenes, bases de datos referenciales, recursos de internet (37). 


\section{VI.A.1.b. Fuentes Terciarias o Generales}

Se trata de documentos que compendian nombres, títulos de revistas y otras publicaciones periódicas, así como nombres de boletines, conferencias y simposios, sitios web, empresas, asociaciones industriales y de diversos servicios, títulos de reportes con información gubernamental; catálogos de libros básicos que contienen referencias y datos bibliográficos y nombres de instituciones nacionales e internacionales al servicio de la investigación. Una fuente terciaria agrupa compendios de fuentes secundarias (37). 


\section{RESULTADOS}

\begin{tabular}{|c|c|c|c|c|}
\hline PAIS & INSTITUCION DE SALUD & $\begin{array}{c}\text { PROFESIONALES } \\
\text { DE SALUD }\end{array}$ & PACIENTE & $\begin{array}{c}\text { INSTRUMENTOS DE } \\
\text { GESTION }\end{array}$ \\
\hline COLOMBIA & $\begin{array}{l}\text { Se aplica el Sistema de Administración para el Sector Salud (SAR) para planear, } \\
\text { hacer, verificar y actuar frente al riesgo. } \\
\text { EPS trabaja en la identificación, la priorización, la mitigación de los riesgos y la } \\
\text { mejora del estado de salud de la población como impacto social. } \\
\text { Se trabajan todas las áreas: riesgos generales en salud, individuales, operativos, } \\
\text { financieros y de mercado. }\end{array}$ & $\begin{array}{l}\text { Reconocimiento del Sistema } \\
\text { de Administración del riesgo y } \\
\text { del plan de implementación. }\end{array}$ & $\begin{array}{l}\text { Grupos priorizados de } \\
\text { riesgo para dirigir las } \\
\text { intervenciones y realizar } \\
\text { seguimiento al impacto en } \\
\text { la gestión del riesgo. }\end{array}$ & $\begin{array}{l}\text { Indicadores de gestión de } \\
\text { riesgos para medir su } \\
\text { impacto. } \\
\text { Formulación de políticas. }\end{array}$ \\
\hline ESPAÑA & $\begin{array}{l}\text { - Creación de Unidades de gestión del riesgo dentro del organigrama hospitalario, } \\
\text { destinadas a canalizar las actividades relacionadas con la seguridad del } \\
\text { paciente. } \\
\text { - Creación de un Programa de gestión de riesgos clínicos. } \\
\text { - Análisis y asesoramiento en las reclamaciones y demandas y en situaciones de } \\
\text { riesgo } \\
\text { - Establecer el nivel de riesgo de cada servicio. } \\
\text { - Ambiente y Sistema para el reporte de eventos adversos. }\end{array}$ & $\begin{array}{l}\text { Formación en gestión de los } \\
\text { riesgos y seguridad del } \\
\text { paciente. } \\
\text { Participación del personal en } \\
\text { todo el proceso de manejo del } \\
\text { riesgo desde la identificación } \\
\text { hasta la evaluación y } \\
\text { retroalimentación. }\end{array}$ & $\begin{array}{l}\text { - Orientación e Información } \\
\text { - al paciente durante su } \\
\text { estancia. } \\
\text { - Consentimiento informado } \\
\text { (preservar la autonomía } \\
\text { del paciente) } \\
\text { - Conocer sus expectativas. }\end{array}$ & $\begin{array}{l}\text { - Guías clínicas } \\
\text { - Reordenación de Servicios } \\
\text { - Uso de indicadores } \\
\text { centinela para minimizar } \\
\text { riesgos. }\end{array}$ \\
\hline ARGENTINA & $\begin{array}{l}\text { - Integrar la acción preventiva en la gestión de la Organización. } \\
\text { - Establecer una vigilancia adecuada de la salud de los trabajadores. } \\
\text { - Crear un Programa de Gestión de Prevención de riesgos clínicos en el } \\
\text { laboratorio clínico para: Identificar, manejar y reducir los peligros/riesgos de } \\
\text { todas las actividades desarrolladas en el Laboratorio Clínico y para adoptar un } \\
\text { conjunto de acciones preventivas para eliminar y/o controlar los riesgos que se } \\
\text { hayan detectado. }\end{array}$ & $\begin{array}{l}\text { Capacitar, asegurando la } \\
\text { participación del personal para } \\
\text { una mejora continua del } \\
\text { desempeño ambiental, de } \\
\text { seguridad y salud laboral. }\end{array}$ & $\begin{array}{l}\text { Laboratorio clínico: Está } \\
\text { conformado tanto por el } \\
\text { personal, los pacientes y } \\
\text { los proveedores, donde se } \\
\text { dan una serie de relaciones } \\
\text { y procesos que requiere } \\
\text { tener un control adecuado } \\
\text { para minimizar los riesgos. }\end{array}$ & $\begin{array}{l}\text { - Plan de Actividades } \\
\text { Preventivas }\end{array}$ \\
\hline$\underline{\text { CHILE }}$ & $\begin{array}{l}\text { - Participación del personal directivo de la Institución. } \\
\text { - Diseño de una Metodología de gestión de riesgo para procesos críticos en } \\
\text { una Institución de Salud Previsional. } \\
\text { - Metodología: Identificación y caracterización del proceso, identificación de } \\
\text { eventos de riesgo, calificación de causas, clasificación del riesgo, diseño de } \\
\text { medidas de mitigación, seguimiento y reporte. } \\
\text { - La metodología se aplicó a tres procesos: Tramitación y pago de solicitudes } \\
\text { de reembolso; mantención de contratos y cobranza de cotizaciones. }\end{array}$ & $\begin{array}{l}\text { Participación del personal } \\
\text { técnico de la Institución en } \\
\text { todo el proceso. }\end{array}$ & $\begin{array}{l}\text { No se involucraron los } \\
\text { clientes, ya que el estudio } \\
\text { fue dirigido a la prevención } \\
\text { de riesgos en los } \\
\text { procesos. }\end{array}$ & $\begin{array}{l}\text { - Ficha para caracterización de } \\
\text { los procesos. } \\
\text { - Ficha de clasificación de } \\
\text { causas. } \\
\text { - Ficha de nivel de riesgos y } \\
\text { medidas adoptadas } \\
\text { - Indicadores que afectan al } \\
\text { usuario e Institución. }\end{array}$ \\
\hline MEXICO & $\begin{array}{l}\text { - Participación de cuerpo directivo en la identificación y gestión del riesgo. } \\
\text { - Estrategias: Reducir errores del personal y mejorar aspectos de la } \\
\text { organización del hospital. } \\
\text { - Niveles de Seguridad: } \\
\text { - Clínico: Seguridad en el proceso de atención, ambiente, insumos, recurso } \\
\text { humano. - Hospitalario: Seguridad de infraestructura.- Del paciente: Buena } \\
\text { praxis, ética médica, seguridad sistema sanitario. -Humano: Acceso a } \\
\text { servicios, seguridad de atención. }\end{array}$ & $\begin{array}{l}\text { Participación de un grupo } \\
\text { interdisciplinario: médicos, } \\
\text { enfermeras, trabajadores } \\
\text { sociales y la gerencia quienes } \\
\text { identificaron, midieron y } \\
\text { evaluaron los factores de } \\
\text { riesgo potenciales para la } \\
\text { seguridad de paciente y de los } \\
\text { propios profesionales. }\end{array}$ & $\begin{array}{l}\text { No se involucra } \\
\text { directamente al paciente, } \\
\text { ya se trabaja en una } \\
\text { metodología para la } \\
\text { medición del riesgo } \\
\text { hospitalario para la } \\
\text { seguridad del paciente. }\end{array}$ & $\begin{array}{l}\text { - Uso del Enfoque Sistémico } \\
\text { (Avedis Donabedian) } \\
\text { - Consulta Metodologías de } \\
\text { gestión del riesgo tanto en la } \\
\text { Administración Pública y de } \\
\text { salud de países } \\
\text { desarrollados. } \\
\text { - Evaluación de variables para } \\
\text { priorizar los riesgos. }\end{array}$ \\
\hline
\end{tabular}

Tabla 6. Cuadro Comparativo: Administración hospitalaria en la Gestión del Riesgo entre países de América Latina y España FUENTE: Elaboración propia 


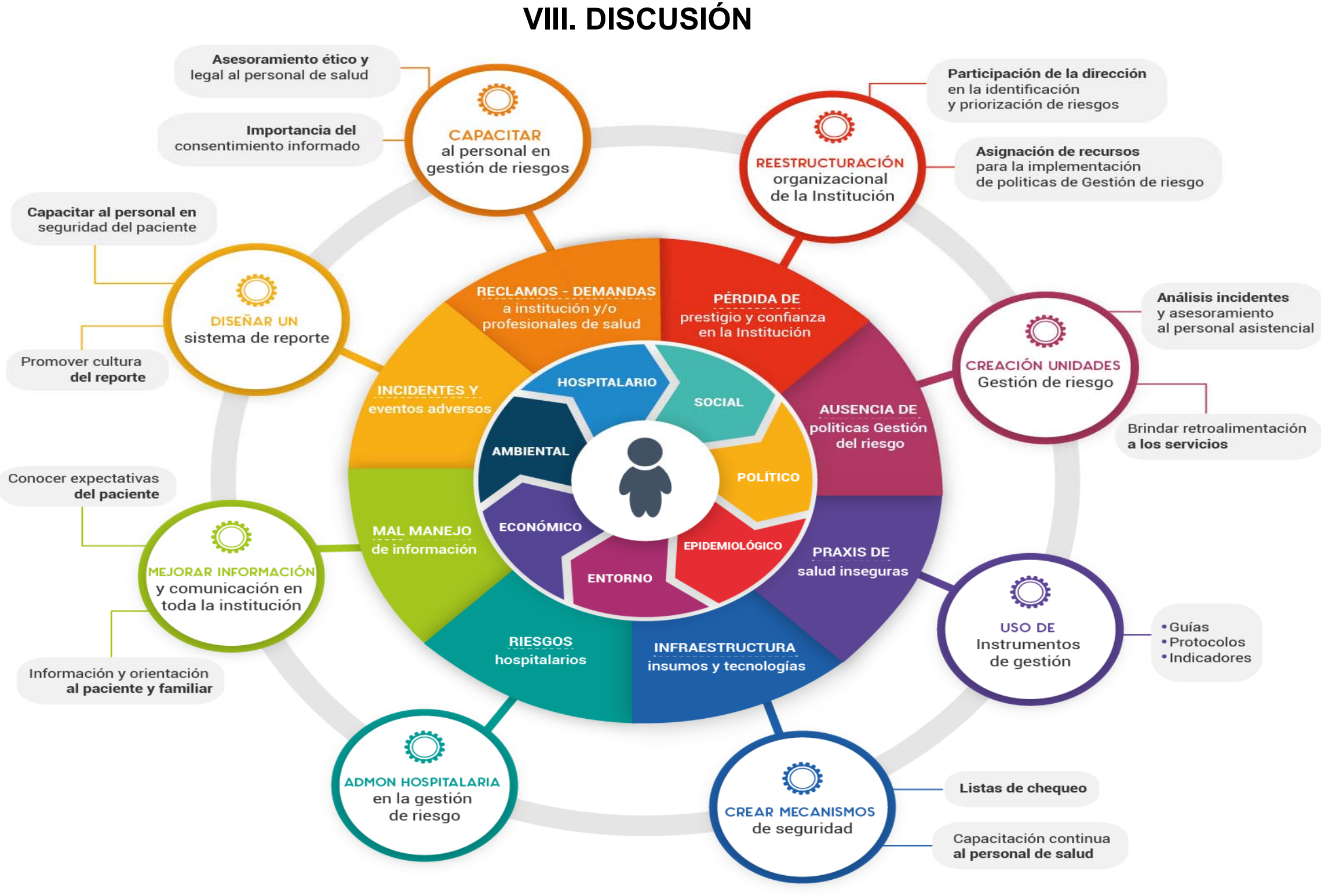

FIGURA 10. Propuesta de un Sistema de Gestión de Riesgo ideal en una Institución de Salud

FUENTE: Elaboración propia 


\section{BIBLIOGRAFÍA}

Agencia de Calidad del SNS. Benchmarking de buenas prácticas en la gestión de riesgos y políticas de reordenación del gobierno clínico en el ámbito hospitalario. Ministerio de Sanidad y Consumo. Madrid, España. 2008. p. 1-99. Disponible en: http://www.msc.es/organizacion/sns/planCalidadSNS/docs/BenchmarkingGestionRiesgos GobiernoClinico.pdf

Alburquerque, C. Artaza, O. Antunes, E. Arteaga, Baffigo, V. Cairo, V. Et al. La transformación de la gestión de hospitales en América Latina y el Caribe. Organización Panamericana de la Salud-

2001. p.353. Disponible en: http://apps.who.int/medicinedocs/documents/s16611s/s16611s.pdf

Aranaz, J. Vitaller B, J. De las complicaciones y efectos adversos a la gestión de los riesgos de la asistencia sanitaria. Edita: Generalitat Valenciana. Consellería de Sanitat. EVES. 2004. p. 1-384.Disponible en: http://proyectoidea.com/publicaciones/DelasComplicacionesyEfectosAdversosalaGestiond eRiesgosdelaAsistencia.pdf

Artaza B, O. Barría I, M. Fuenzalida, A, Nuñez, K. Quintana, A. Vargas,I. Nevegas, C. Vidales, A. Modelo de Gestión de Establecimientos Hospitalarios. Ministerio de Salud. Chile. p.1-88. Disponible en: http://www.bibliotecaminsal.cl/wp/wp-content/uploads/2016/03/9.pdf

Departamento Administrativo de la Función Pública. Guía para la administración del riesgo. Cuarta edición. Bogotá D.C., Colombia. 2011. p. 1-52. Disponible en: http://www.funcionpublica.gov.co/documents/418537/506911/1592.pdf/73e5a159-2d8f41aa-8182-eb99e8c4f3ba

Ministerio de Salud y Protección Social. Gestión Integral del Riesgo en Salud. Perspectiva desde el Aseguramiento en el contexto del Modelo Integral de Atención en Salud. Módulo 7. Bogotá D.C., Colombia. 2015. P. 1-39. Disponible en: https://www.minsalud.gov.co/sites/rid/Lists/BibliotecaDigital/RIDE/VP/DOA/Perspectivaaseguramiento-comite-ampliado.pdf

Organización Mundial de la Salud-OMS. Informe sobre la salud en el mundo. Reducir los riesgos y promover una vida sana. Francia. 2002. P 1-165. Disponible en: http://www.who.int/whr/2002/es/

Puerto J, D. La Gestión del riesgo en Salud en Colombia. [Internet]. Universidad Nacional de Colombia. Facultad de Ciencias Económicas. Bogotá D.C., Colombia. 2011. Disponible en: http://www.bdigital.unal.edu.co/7023/1/940622.2011.pdf

Universidad Santo Tomás. Guía para trabajos de Grado. Aplicando estilo Vancouver.2012.p. 1-22. 


\section{REFERENCIAS BIBLIOGRAFICAS}

${ }^{1}$ Alburquerque, C. Artaza, O. Antunes, E. Arteaga, Baffigo, V. Cairo, V. Et al. La transformación de la gestión de hospitales en América Latina y el Caribe. Organización Panamericana de la Salud- OPS. Washington. $2001 . \quad$ p.353. Disponible en: http://apps.who.int/medicinedocs/documents/s16611s/s16611s.pdf

${ }^{2}$ Pardo, A. Navarro, C. Arguedas, R.Albeniz, C. Morón,J. Barreras y retos de las Unidades funcionales de gestión de riesgos en los hospitales del Servicio Madrileño de Salud. Revista de Calidad Asistencial ELSEVIER. [Internet]. 2014. Marzo-Abril. Vol.29 (2): p. 84-91. Disponible en: http://www.elsevier.es/es-revista-revista-calidad-asistencial-256sumario-vol-29-num-2-S1134282X14X00035

${ }^{3}$ Pardo, A. Navarro, C. Arguedas, R.Albeniz, C. Morón,J. Barreras y retos de las Unidades funcionales de gestión de riesgos en los hospitales del Servicio Madrileño de Salud. Revista de Calidad Asistencial ELSEVIER. [Internet]. 2014. Marzo-Abril. Vol.29 (2): p. 84-91. Disponible en: http://www.elsevier.es/es-revista-revista-calidad-asistencial-256sumario-vol-29-num-2-S1134282X14X00035

${ }^{4}$ Organización Mundial de la Salud-OMS. Informe sobre la salud en el mundo. Reducir los riesgos y promover una vida sana. Francia. 2002. P 1-165. Disponible en: http://www.who.int/whr/2002/es/

${ }^{5}$ Martínez FJ, Ruiz Ortega, JM. Manual de Gestión de Riesgos Sanitarios. Madrid, España. Editorial Díaz de Santos. 2001. p 59. Disponible en: https://books.google.com.co/books?id=NKEcHZWJYkC\&printsec=frontcover\&hl=es\&source=gbs ge summary $r \& c a d=0 \# v=0 n e p$ age\&q\&f=false

${ }^{6}$ Martínez FJ, Ruiz Ortega, JM. Manual de Gestión de Riesgos Sanitarios. Madrid, España. Editorial Díaz de Santos. 2001. p 60. Disponible en: https://books.google.com.co/books?id=NKEcHZWJYkC\&printsec=frontcover\&hl=es\&source=gbs ge summary $r \& c a d=0 \# v=0 n e p$ age\&q\&f=false

7 Martínez FJ, Ruiz Ortega, JM. Manual de Gestión de Riesgos Sanitarios. Madrid, España. Editorial Díaz de Santos. 2001. p 57. Disponible en: https://books.google.com.co/books?id=NKEcHZWJYkC\&printsec=frontcover\&hl=es\&source=gbs ge summary $r \& c a d=0 \# v=o n e p$ $\underline{\text { age } \& q \& f=f a l s e}$

${ }^{8}$ Castillo P, R. La administración de los servicios de salud.[Internet]. Disponible en: http://www.monografias.com/trabajos96/la-administracion-servicios-salud/laadministracion-servicios-salud.shtml

${ }^{9}$ Concepto de Gestión. Disponible en: http://concepto.de/gestion/\#ixzz5ENg2rB6b 
${ }^{10}$ Pacheco S, Hugo E. Gestión, tipos, gestión investigativa, enfoque. Junio. 2013. Disponible en: http://doctxs6.blogspot.com.co/2013/01/gestion-tipos-gestion-investigativa 27.html

11 Casassus, Juan. Problemas de la gestión educativa en América Latina (la tensión entre los paradigmas de tipo A y el tipo B). Octubre. 2000. p.1-27. Disponible en: http://www.lie.upn.mx/docs/Especializacion/Gestion/Lec2\%20.pdf

12 Departamento Administrativo de la Función Pública. Guía para la Administración del riesgo. Cuarta Edición. Bogotá D.C, Colombia. Septiembre. $52 . \quad$ Disponible en: http://www.funcionpublica.gov.co/documents/418537/506911/1592.pdf/73e5a159-2d8f41aa-8182-eb99e8c4f3ba

${ }^{13}$ Comunidad de Madrid. Análisis de riesgos. Gestión de Riesgos. p. 1-7. Disponible en: http://www.madrid.org/cs/StaticFiles/Emprendedores/Analisis Riesgos/pages/pdf/meto dologia/2GestiondeRiesgos(AR) es.pdf

${ }^{14}$ Oliveira, Wallace. ¿Qué es la gestión de riesgos? Propósito y concepto. Julio. 2017. Disponible en https://www.heflo.com/es/blog/gestion-de-riesgos/que-es-gestion-de-riesgos/

15 Encolombia. Gestión del Riesgo en las Instalaciones de salud. Disponible en: https://encolombia.com/medicina/guiasmed/emerg-hospitalarias/capitulo5 gestiondelriesgoeninstalaciones/

16 Álvarez U, G. Roca G, S.Chaux D, M.Pineda G, F. Implementación del Sistema de Administración de Riesgos -SAR por Entidades Promotoras de Salud del Régimen Contributivo en Colombia 2008-2011. Revista Monitor Estratégico [Internet]. 2012. Enero-Junio;(1). p. 6-14.Disponible en: https://docs.supersalud.gov.co/PortalWeb/Comunicaciones/MonitorEstrategico/MonitorEstrategico001-2012\%20.pdf

17 Ministerio de Protección Social. Resolución 1740 de 2008. Por la cual se dictan disposiciones relacionadas con el Sistema de Administración de Riesgos para las entidades Promotoras de Salud del Régimen Contributivo y Entidades Adaptadas previstas en el Sistema de Habilitación, condiciones financieras y de suficiencia patrimonial se definen las fases para su implementación

se dictan otras disposiciones. Disponible en:
https://www.minsalud.gov.co/sites/rid/Lists/BibliotecaDigital/RIDE/DE/DIJ/Resoluci\%C3 \%B3n 1740 de 2008.pdf

18 Álvarez U, G. Roca G, S.Chaux D, M.Pineda G, F. Implementación del Sistema de Administración de Riesgos -SAR por Entidades Promotoras de Salud del Régimen Contributivo en Colombia 2008-2011. Revista Monitor Estratégico [Internet].2012. EneroJunio;(1). p. 6-14. $\quad$ Disponible https://docs.supersalud.gov.co/PortalWeb/Comunicaciones/MonitorEstrategico/MonitorEstrategico001-2012\%20.pdf 
19 Álvarez U, G. Roca G, S.Chaux D, M.Pineda G, F. Implementación del Sistema de Administración de Riesgos -SAR por Entidades Promotoras de Salud del Régimen Contributivo en Colombia 2008-2011. Revista Monitor Estratégico [Internet]. 2012.

Enero-Junio;(1). $\quad$ p. 6-14. Disponible https://docs.supersalud.gov.co/PortalWeb/Comunicaciones/MonitorEstrategico/MonitorEstrategico001-2012\%20.pdf

20 Álvarez U, G. Roca G, S.Chaux D, M.Pineda G, F. Implementación del Sistema de Administración de Riesgos -SAR por Entidades Promotoras de Salud del Régimen Contributivo en Colombia 2008-2011. Revista Monitor Estratégico [Internet]. 2012. Enero-Junio;(1). $\quad$ p. 6-14. Disponible https://docs.supersalud.gov.co/PortalWeb/Comunicaciones/MonitorEstrategico/MonitorEstrategico001-2012\%20.pdf

${ }^{21}$ Rosado B, L. Martínez S, J. Gestión de riesgos desde la visión de la gestión hospitalaria. Revista de Calidad Asistencial ELSEVIER [Internet]. 2005. Marzo. Vol. 20 (2): p. 110114. Disponible en: http://www.elsevier.es/es-revista-revista-calidad-asistencial-256articulo-gestion-riesgos-desde-vision-gestion-13073200

22 Pardo, z. Jara, A. Menchen, B. Padilla, D.Martín, J. Hernández, J. Et al. Puesta en marcha de una Unidad de Gestión de Riesgos Clínicos Hospitalaria. Revista de Calidad Asistencial ELSEVIER. [Internet]. 2005. Junio- Vol.20 (4): p. 211-215. Disponible en: http://www.elsevier.es/es-revista-revista-calidad-asistencial-256-articulo-puestamarcha-una-unidad-gestion-13075821

${ }^{23}$ Pardo, z. Jara, A. Menchen, B. Padilla, D.Martín, J. Hernández, J. Et al. Puesta en marcha de una Unidad de Gestión de Riesgos Clínicos Hospitalaria. Revista de Calidad Asistencial ELSEVIER. [Internet]. 2005. Junio- Vol.20 (4): p. 211-215. Disponible en: http://www.elsevier.es/es-revista-revista-calidad-asistencial-256-articulo-puestamarcha-una-unidad-gestion-13075821

${ }^{24}$ Pardo, z. Jara, A. Menchen, B. Padilla, D.Martín, J. Hernández, J. Et al. Puesta en marcha de una Unidad de Gestión de Riesgos Clínicos Hospitalaria. Revista de Calidad Asistencial ELSEVIER. [Internet]. 2005. Junio- Vol.20 (4): p. 211-215. Disponible en: http://www.elsevier.es/es-revista-revista-calidad-asistencial-256-articulo-puestamarcha-una-unidad-gestion-13075821

${ }^{25}$ Pardo, z. Jara, A. Menchen, B. Padilla, D.Martín, J. Hernández, J. Et al. Puesta en marcha de una Unidad de Gestión de Riesgos Clínicos Hospitalaria. Revista de Calidad Asistencial ELSEVIER. [Internet]. 2005. Junio- Vol.20 (4): p. 211-215. Disponible en: http://www.elsevier.es/es-revista-revista-calidad-asistencial-256-articulo-puestamarcha-una-unidad-gestion-13075821

${ }^{25}$ Dear P, S. Importancia de la gestión de seguridad en el laboratorio clínico de un hospital materno infantil. Revista del Hospital Materno Infantil Ramón Sardá [Internet]. 2008. Vol.27 (4): p. 120-123. Fecha de consulta:10 de mayo de 2018. Disponible en: http://www.redalyc.org/articulo.oa?id=91227305. ISSN 1514-9838 
${ }^{26}$ Dear P, S. Importancia de la gestión de seguridad en el laboratorio clínico de un hospital materno infantil. Revista del Hospital Materno Infantil Ramón Sardá [Internet]. 2008. Vol.27 (4): p. 120-123. Fecha de consulta:10 de mayo de 2018. Disponible en: http://www.redalyc.org/articulo.oa?id=91227305. ISSN 1514-9838

27 Torres N, C.Malta C,N.Zapata C, C. Aburto V, Víctor.. Metodología de Gestión de Riesgo para procesos en una Institución de Salud Previsional. Revista Universidad, Ciencia y Tecnología [Internet]. 2015. Junio. Vol.19 (75). p. 98 - 109. Disponible en: http://www.scielo.org.ve/scielo.php?script=sci issuetoc\&pid=1316482120150002\&lng=es\&nrm=iso

28 Torres N, C.Malta C,N.Zapata C, C. Aburto V, Víctor. Metodología de Gestión de Riesgo para procesos en una Institución de Salud Previsional. Revista Universidad, Ciencia y Tecnología [Internet]. 2015. Junio. Vol.19 (75). p. 98 - 109. Disponible en: http://www.scielo.org.ve/scielo.php?script=sci issuetoc\&pid=1316$\underline{482120150002 \& \operatorname{lng}=e s \& n r m=i s o}$

29 Torres N, C.Malta C,N.Zapata C, C. Aburto V, Víctor. Metodología de Gestión de Riesgo para procesos en una Institución de Salud Previsional. Revista Universidad, Ciencia y Tecnología [Internet]. 2015. Junio. Vol.19 (75). p. 98 - 109. Disponible en: http://www.scielo.org.ve/scielo.php?script=sci issuetoc\&pid=1316$\underline{482120150002 \& \operatorname{lng}=e s \& n r m=i s o}$

30 Torres N, C.Malta C,N.Zapata C, C. Aburto V, Víctor. Metodología de Gestión de Riesgo para procesos en una Institución de Salud Previsional. Revista Universidad, Ciencia y Tecnología [Internet]. 2015. Junio. Vol.19 (75). p. 98 - 109. Disponible en: http://www.scielo.org.ve/scielo.php?script=sci issuetoc $\&$ pid=1316$\underline{482120150002 \& \operatorname{lng}=e s \& n r m=i s o}$

30 Torres N, C.Malta C,N.Zapata C, C. Aburto V, Víctor. Metodología de Gestión de Riesgo para procesos en una Institución de Salud Previsional. Revista Universidad, Ciencia y Tecnología [Internet]. 2015. Junio. Vol.19 (75). p. 98 - 109. Disponible en: http://www.scielo.org.ve/scielo.php?script=sci issuetoc\&pid=1316$\underline{482120150002 \& \operatorname{lng}=e s \& n r m=i s o}$

${ }^{31}$ Santacruz V, J. Hernández T, F. Fajardo D, G. Evaluación del riesgo para la seguridad del paciente en Establecimientos de Salud. Red de Revistas Científicas de América Latina, el Caribe, España y Portugal. Cirugía y Cirujanos [Internet]. 2010. NoviembreDiciembre. $\quad 78 . \quad$ p. $514 \quad 516 . \quad$ Disponible $\quad$ en: http://www.redalyc.org/articulo.oa?id=66220323009.ISSN 0009-7411

${ }^{32}$ Santacruz V, J. Hernández T, F. Fajardo D, G. Evaluación del riesgo para la seguridad del paciente en Establecimientos de Salud. Red de Revistas Científicas de América Latina, el Caribe, España y Portugal. Cirugía y Cirujanos [Internet]. 2010. NoviembreDiciembre. $\quad 78 . \quad$ p. $514 \quad-516 . \quad$ Disponible $\quad$ en: http://www.redalyc.org/articulo.oa?id=66220323009 .ISSN 0009-7411 
${ }^{33}$ Santacruz V, J. Hernández T, F. Fajardo D, G. Evaluación del riesgo para la seguridad del paciente en Establecimientos de Salud. Red de Revistas Científicas de América Latina, el Caribe, España y Portugal. Cirugía y Cirujanos [Internet]. 2010. NoviembreDiciembre. $\quad 78 . \quad$ p. $\quad 514 \quad-516 . \quad$ Disponible en: http://www.redalyc.org/articulo.oa?id=66220323009 .ISSN 0009-7411

${ }^{34}$ Hernández S, Roberto. Hernández Collado, Carlos. Baptista L, Pilar. Metodología de la Investigación. Cuarta Edición. Editorial Mac Graw-Hill Interamericana. México D.F. 2006. P.65-67.

${ }^{35}$ MerinoT, Alejandra. Como escribir documentos científicos (Parte 3). Artículo de revisión. Salud en Tabasco.Secretaria de Salud del Estado de Tabasco Villahermosa México. [Internet]. 2001. Enero - Agosto. Vol. 17. (1-2). p. 36-40 Disponible en: http://www.redalyc.org/pdf/487/48721182006.pdf

${ }^{36}$ Gallego L, Josefa. Junca C. Manuela. Fuentes y Servicios de Información. Universitat Oberta de Catalunya. Primera Edición. Barcelona- España. Septiembre. 2009. $p \quad 8-9, \quad p \quad 19 \quad-21 . \quad$ Disponible $\quad$ en: http://openaccess.uoc.edu/webapps/o2/bitstream/10609/241/5/Fuentes\%20de\%20infor maci\%C3\%B3n\%20I M\%C3\%B3dulo\%201 Fuentes\%20y\%20servicios\%20de\%20info rmaci\%C3\%B3n.pdf. ISBN: 978-84-692-4639-9

${ }^{37}$ Hernández S, Roberto. Hernández Collado, Carlos. Baptista L, Pilar. Metodología de la Investigación. Cuarta Edición. Editorial Mac Graw-Hill Interamericana. México D.F. 2006. P.65-67. 